\title{
ANTICHAINS IN WEIGHT POSETS ASSOCIATED WITH GRADINGS OF SIMPLE LIE ALGEBRAS
}

\author{
DMITRI I. PANYUSHEV
}

\begin{abstract}
For a reductive Lie algebra $\mathfrak{h}$ and a simple finite-dimensional $\mathfrak{h}$-module $V$, the set of weights of $V, \mathcal{P}(V)$, is equipped with a natural partial order. We consider antichains in the weight poset $\mathcal{P}(V)$ and a certain operator $\mathfrak{X}$ acting on antichains. Eventually, we impose stronger constraints on $(\mathfrak{h}, V)$ and stick to the case in which $\mathfrak{h}=\mathfrak{g}(0)$ and $V=\mathfrak{g}(1)$ for a $\mathbb{Z}$-grading $\mathfrak{g}=\bigoplus_{i \in \mathbb{Z}} \mathfrak{g}(i)$ of a simple Lie algebra $\mathfrak{g}$. Then $V$ is a weight multiplicity free $\mathfrak{h}$-module and $\mathcal{P}(V)$ can be regarded as a subposet of $\Delta^{+}$, where $\Delta$ is the root system of $\mathfrak{g}$. Our goal is to demonstrate that the weight posets associated with $\mathbb{Z}$-gradings exhibit many good properties that are similar to those of $\Delta^{+}$that are observed earlier in [14].
\end{abstract}

\section{CONTENTS}

1. Introduction 1

2. Posets, weight posets and gradings 4

3. Antichains and upper ideals in the abelian case 10

4. Antichains and upper ideals in the extra-special case 14

5. Conjectures and examples 21

$\begin{array}{ll}\text { References } & 27\end{array}$

\section{INTRODUCTION}

Let $(\mathcal{P}, \preccurlyeq)$ be a finite poset and $\mathfrak{A n}(\mathcal{P})$ the set of all antichains in $\mathcal{P}$. If $\Gamma \in \mathfrak{A} \mathfrak{n}(\mathcal{P})$, then

$$
\begin{gathered}
I_{+}(\Gamma)=I(\Gamma):=\{\nu \in \mathcal{P} \mid \gamma \preccurlyeq \nu \text { for some } \gamma \in \Gamma\} \text { and } \\
I_{-}(\Gamma):=\{\nu \in \mathcal{P} \mid \nu \preccurlyeq \gamma \text { for some } \gamma \in \Gamma\}
\end{gathered}
$$

We say that $I(\Gamma)$ (resp. $I_{-}(\Gamma)$ ) is the upper (resp. lower) ideal of $\mathcal{P}$ generated by $\Gamma$. For any $M \subset \mathcal{P}$, let $\min (M)($ resp. $\max (M))$ denote the set of minimal (resp. maximal) elements of $M$ with respect to ' $\preccurlyeq$ '. Then $\Gamma=\min (I(\Gamma))=\max \left(I_{-}(\Gamma)\right)=I(\Gamma) \cap I_{-}(\Gamma)$ and the above formulae provide one-to-one correspondences between the antichains, upper ideals, and lower ideals of $\mathcal{P}$.

2010 Mathematics Subject Classification. 06A07, 17B20, $20 \mathrm{~F} 55$.

Key words and phrases. Graded poset, root system, graded Lie algebra. 
Definition 1. The reverse operator associated with $\mathcal{P}, \mathfrak{X}=\mathfrak{X}_{\mathcal{P}}: \mathfrak{A} \mathfrak{n}(\mathcal{P}) \rightarrow \mathfrak{A} \mathfrak{n}(\mathcal{P})$, is defined by $\mathfrak{X}(\Gamma)=\max (\mathcal{P} \backslash I(\Gamma))$.

It is easily seen that $\mathfrak{X}\left(\min \left(\mathcal{P} \backslash I_{-}(\Gamma)\right)\right)=\Gamma$, hence $\mathfrak{X}$ is invertible. The order of $\mathfrak{X}$, denoted $\operatorname{ord}(\mathfrak{X})$, is the order of the cyclic group $\langle\mathfrak{X}\rangle$ generated by $\mathfrak{X}$ in the permutation group on $\mathfrak{A} \mathfrak{n}(\mathcal{P})$. By definition, an $\mathfrak{X}$-orbit is an orbit of the group $\langle\mathfrak{X}\rangle$ in $\mathfrak{A} \mathfrak{n}(\mathcal{P})$. The early history of the reverse operators, which goes back to 1970's, is related to the special case in which $\mathcal{P}$ is the Boolean algebra $\mathbb{B}^{n}$, see [7] and references therein. The study of $\mathfrak{X}$ in the general setting was initiated in $[9,5]$.

For a natural class of posets $\mathcal{P}$, we wish to know $\# \mathfrak{A n}(\mathcal{P}), \max _{\Gamma \in \mathfrak{A} \mathfrak{n}(\mathcal{P})}(\# \Gamma)$, ord $(\mathfrak{X})$, and other properties of the $\mathfrak{X}$-orbits in $\mathfrak{A} \mathfrak{n}(\mathcal{P})$. It is also of interest to determine the following refinements ( $t$-analogues) of $\# \mathfrak{A} \mathfrak{n}(\mathcal{P})$ :

1) Set $\mathcal{N}_{\mathcal{P}}(t)=\sum_{\Gamma \in \mathfrak{A}(\mathcal{P})} t^{\# \Gamma}=\sum N_{i} t^{i}$. Here the coefficient of $t^{i}$ is the number of antichains of size $i$. Consequently, $\mathcal{N}_{\mathcal{P}}^{\prime}(1) / \mathcal{N}_{\mathcal{P}}(1)$ is the average value of the size of all antichains in $\mathcal{P}$. It is clear that $N_{1}=\# \mathcal{P}$ and $\operatorname{deg} \mathcal{N}_{\mathcal{P}}(t)=\max _{\Gamma \in \mathfrak{A} \mathfrak{n}(\mathcal{P})}(\# \Gamma)$.

2) Set $\mathcal{M}_{\mathcal{P}}(t)=\sum_{\Gamma \in \mathfrak{A}(\mathcal{P})} t^{\# I(\Gamma)}=\sum M_{i} t^{i}$. Here the coefficient of $t^{i}$ is the number of upper ideals of cardinality $i$. Consequently, $\mathcal{M}_{\mathcal{P}}^{\prime}(1) / \mathcal{M}_{\mathcal{P}}(1)$ is the average value of the cardinality of all upper ideals. It is clear that $\mathcal{M}_{\mathcal{P}}(t)$ is monic and $\operatorname{deg} \mathcal{M}_{\mathcal{P}}(t)=\# \mathcal{P}$.

These two $t$-analogues of $\mathfrak{A n}(\mathcal{P})$ will be referred to as the $\mathcal{M}$ - and $\mathcal{N}$-polynomials of $\mathcal{P}$.

Let $\Delta^{+}$be a set of positive roots of a reduced irreducible root system $\Delta$. Then $\Delta^{+}$is a graded poset, and there is a number of striking results on $\mathfrak{A} \mathfrak{n}\left(\Delta^{+}\right)$and $\mathfrak{X}_{\Delta^{+}}$. We refer to $[3,11]$ for basic definitions and properties of root systems. Let $\Pi=\left\{\alpha_{1}, \ldots, \alpha_{n}\right\}$ be the set of simple roots in $\Delta^{+}, W$ the Weyl group, and $h$ the Coxeter number of $\Delta$. The partial order in $\Delta^{+}$is defined by the condition that $\gamma$ covers $\mu$ if and only if $\gamma-\mu \in \Pi$. For $\gamma \in \Delta$, let $\left[\gamma: \alpha_{i}\right]$ be the coefficient of $\alpha_{i}$ in the expression of $\gamma$ via the simple roots. The height of $\gamma$ is $\operatorname{ht}(\gamma)=\sum_{i=1}^{n}\left[\gamma: \alpha_{i}\right]$. Then

- $\min \left(\Delta^{+}\right)=\Pi$ and $\max \left(\Delta^{+}\right)=\{\theta\}$, where $\theta$ is the highest root;

- $\Delta^{+}$is graded, with rank function $\gamma \mapsto \operatorname{ht}(\gamma)$. Recall that $1 \leqslant \mathrm{ht}(\gamma) \leqslant h-1$;

- $\# \mathfrak{A} \mathfrak{n}\left(\Delta^{+}\right)=\prod_{i=1}^{n} \frac{h+m_{i}+1}{m_{i}+1}$, where $m_{1}, \ldots, m_{n}$ are the exponents of $W$, see [6].

- if $\Delta$ is of type $\mathbf{A}_{n}$, then $\# \mathfrak{A n}\left(\Delta^{+}\right)$is the $(n+1)$-th Catalan number and the coefficients of $\mathcal{N}_{\Delta^{+}}(t)$ are the Narayana numbers. No general uniform expression for $\mathcal{N}_{\Delta^{+}}(t)$ is known; but case-by-case computations show that $\mathcal{N}_{\Delta^{+}}(t)$ is always palindromic and unimodal.

It was conjectured in [14, Conjecture 2.1] that $\mathfrak{X}_{\Delta^{+}}$satisfies the following properties:

(i) $\left(\mathfrak{X}_{\Delta^{+}}\right)^{h}$ is the permutation on $\mathfrak{A n}\left(\Delta^{+}\right)$induced by $-w_{0}$, where $w_{0} \in W$ is the longest element $\left(=\right.$ unique element taking $\Delta^{+}$to $\left.-\Delta^{+}\right)$. In particular, ord $\left(\mathfrak{X}_{\Delta^{+}}\right) \in$ $\{h, 2 h\}$ and $\operatorname{ord}\left(\mathfrak{X}_{\Delta^{+}}\right)=h$ if and only if $w_{0}=-1$;

(ii) Let $\mathcal{O}$ be an $\mathfrak{X}_{\Delta^{+}}$-orbit in $\mathfrak{A n}\left(\Delta^{+}\right)$. Then the average value $\left(\sum_{\Gamma \in \mathcal{O}} \# \Gamma\right) / \# \mathcal{O}$ does not depend on $\mathcal{O}$ and equals $\#\left(\Delta^{+}\right) / h=n / 2$. 
This conjecture has been proved in [2]. However, similar conjectures in [14] for several related graded posets (e.g. for $\mathcal{P}=\Delta^{+} \backslash \Pi$ ) are still open.

We are going to describe a natural class of posets having the similar properties. As there is a simple Lie algebra behind $\Delta$, it is natural to explore posets related to representations of semisimple (reductive) Lie algebras. Let $\mathcal{P}(V)$ be the set of weights of an irreducible finite-dimensional representation $V$ of a reductive Lie algebra. Then $\mathcal{P}(V)$ is a graded poset. We show that $\mathcal{P}(V) \simeq \mathcal{P}\left(V^{*}\right)$ and $\mathfrak{X}_{\mathcal{P}(V)}$ can naturally be written as a product of two involutions, see Remark 2.3. Another promising observation is that if $V$ is weight multiplicity free (=wmf), then $\mathcal{P}(V)$ is rank symmetric, rank unimodal, and Sperner (see precise definitions in Section 2). However, the class of all (or even wmf) weight posets is too large for interesting properties, and we stick to wmf representations associated with $\mathbb{Z}$-gradings of a simple Lie algebra $\mathfrak{g}$. Given a $\mathbb{Z}$-grading $\mathfrak{g}=\bigoplus_{i \in \mathbb{Z}} \mathfrak{g}(i)$, we are interested in the weight poset, $\Delta(1)$, of the $\mathfrak{g}(0)$-module $\mathfrak{g}(1)$. Assuming that $\Delta$ is the root system of $\mathfrak{g}$, we may regard $\Delta(1)$ as a subposet of $\Delta^{+}$. Our aim is to demonstrate that the posets of the form $\Delta(1)$ exhibit many good properties that are akin to the above properties of $\Delta^{+}$. The basic material on gradings of $\mathfrak{g}$ is gathered in Section 2.2.

We consider in details two simplest classes of $\mathbb{Z}$-gradings:

- the abelian gradings, $\mathfrak{g}=\mathfrak{g}(-1) \oplus \mathfrak{g}(0) \oplus \mathfrak{g}(1)$ (Section 3);

- the extra-special gradings, $\mathfrak{g}=\bigoplus_{j=-2}^{2} \mathfrak{g}(j)$ \& $\operatorname{dim} \mathfrak{g}(2)=1$ (Section 4 ).

To a great extent, our results for them are similar, but the proofs become more tricky in the extra-special case. Using the Kostant-Macdonald identity [12, Cor. 2.5], we prove that $\mathcal{M}_{\Delta(1)}(t)=\prod_{\gamma \in \Delta(1)} \frac{1-t^{\mathrm{ht}(\gamma)+1}}{1-t^{\mathrm{ht}(\gamma)}}$ (Theorems 3.3 and 4.4); in particular, $\# \mathfrak{A} \mathfrak{n}(\Delta(1))=$ $\prod_{\gamma \in \Delta(1)} \frac{\mathrm{ht}(\gamma)+1}{\mathrm{ht}(\gamma)}$. All the respective $\mathcal{N}$-polynomials are computed, too. A relationship between $\mathfrak{A} \mathfrak{n}(\Delta(1))$ and certain elements of the Weyl group $W$ of $\mathfrak{g}$ is established (Theorems 3.1 and 4.2). We also provide a model of the poset $\mathfrak{A} \mathfrak{n}(\Delta(1))$ related to the weight poset of a certain representation of the dual Lie algebra $\mathfrak{g}^{\vee}$, see Theorems 3.6 and 4.10. Yet certain nice features of the extra-special case have no 'abelian' analogues. For instance, we have $\# \mathfrak{A} \mathfrak{n}(\Delta(1))=\# \Pi_{l} \cdot(h-1)$, where $\Pi_{l}$ is the set of long simple roots; $\mathcal{M}_{\Delta(1)}(t)$ is closely related to the Lusztig $t$-analogue of the zero weight multiplicity for the representation of $\mathfrak{g}^{\vee}$ with highest weight $\theta^{\vee}$, see Remark 4.6; $\operatorname{deg} \mathcal{N}_{\Delta(1)}(t) \leqslant 3$ (Theorem 4.7) and there is a nice explicit formula for $\mathcal{N}_{\Delta(1)}(t)$ if $\mathfrak{g}$ is of type $\operatorname{ADE}$ (Corollary 4.9).

Section 5 contains numerous examples and our general conjectures on $\mathfrak{A} \mathfrak{n}(\Delta(1))$ and $\mathfrak{X}_{\Delta(1)}$. In particular, we conjecture that

(1) our formula for $\mathcal{M}_{\Delta(1)}(t)$, which us proved in the abelian and extra-special cases, remains valid for all $\mathbb{Z}$-gradings of $\mathfrak{g}$;

(2) if $\mathfrak{g}(1)$ is a simple $\mathfrak{g}(0)$-module, then ord $\left(\mathfrak{X}_{\Delta(1)}\right)$ equals $\left(\max _{\gamma \in \Delta(1)}\right.$ ht $\left.(\gamma)\right)+1$;

(3) if $\mathcal{O}$ is an $\mathfrak{X}_{\Delta(1)}$-orbit in $\mathfrak{A} \mathfrak{n}(\Delta(1))$, then the average value $\left(\sum_{\Gamma \in \mathcal{O}} \# \Gamma\right) / \# \mathcal{O}$ does not depend on $\mathcal{O}$ and equals $\# \Delta(1) / \operatorname{ord}\left(\mathfrak{X}_{\Delta(1)}\right.$; 
(4) if $\mathcal{O}$ is an $\mathfrak{X}_{\Delta(1)}$-orbit in $\mathfrak{A} \mathfrak{n}(\Delta(1))$, then the average value $\left(\sum_{\Gamma \in \mathcal{O}} \# I(\Gamma)\right) / \# \mathcal{O}$ does not depend on $\mathcal{O}$ and equals $\# \Delta(1) / 2$.

(5) the polynomial $\mathcal{N}_{\Delta(1)}(t)$ is palindromic if and only if $\Delta(1)$ has a unique rank level of maximal size.

Our examples show that properties (1)-(3),(5) fail for some wmf representations that are not related to $\mathbb{Z}$-gradings. We also touch upon some aspects of the " $t=-1$ phenomenon" [24] related to the posets $\Delta(1)$ and their $\mathcal{M}$-polynomials.

In a subsequent article, we develop some general theory related to the weight posets $\Delta(1)$ and discuss manifestations of the cyclic sieving phenomenon [22] in this setting.

\section{Posets, Weight POSETS AND GRADINGS}

We begin with recalling some notation and standard facts on posets, see [26, Ch. 3]. The Hasse diagram of $\mathcal{P}$ is the directed graph $\mathcal{H}(\mathcal{P})$ whose vertex set is $\mathcal{P}$ and the set of edges is $\left\{\left(x, x^{\prime}\right) \in \mathcal{P} \times \mathcal{P} \mid x\right.$ covers $\left.x^{\prime}\right\}$. (Such an edge is depicted by $\underset{x^{\prime}}{\longrightarrow}$.) Then $\mathcal{P}$ is the disjoint union of subposets $\mathcal{P}_{1}$ and $\mathcal{P}_{2}$ (denoted $\mathcal{P}=\mathcal{P}_{1} \sqcup \mathcal{P}_{2}$ ), if $\mathcal{H}(\mathcal{P})$ is the disjoint union of graphs $\mathcal{H}\left(\mathcal{P}_{1}\right)$ and $\mathcal{H}\left(\mathcal{P}_{2}\right)$. A poset is said to be connected if it is not a disjoint union of two nonempty subposets. The following easy observation reduces many problems that are of interest for us to the case of connected posets.

Lemma 2.1. If $\mathcal{P}=\mathcal{P}_{1} \sqcup \mathcal{P}_{2}$, then $\mathcal{M}_{\mathcal{P}}(t)=\mathcal{M}_{\mathcal{P}_{1}}(t) \cdot \mathcal{M}_{\mathcal{P}_{2}}(t), \mathcal{N}_{\mathcal{P}}(t)=\mathcal{N}_{\mathcal{P}_{1}}(t) \cdot \mathcal{N}_{\mathcal{P}_{2}}(t)$, and $\operatorname{ord}\left(\mathfrak{X}_{\mathcal{P}}\right)=$ I.c.m. $\left\{\operatorname{ord}\left(\mathfrak{X}_{\mathcal{P}_{1}}\right), \operatorname{ord}\left(\mathfrak{X}_{\mathcal{P}_{2}}\right)\right\}$.

A poset $\mathcal{P}$ is graded if it admits a rank function. A rank function on $\mathcal{P}$ is a map $r: \mathcal{P} \rightarrow \mathbb{N}$ such that $r(x)=r(y)+1$ whenever $x$ covers $y$.

As is well known, $\mathfrak{A} \mathfrak{n}(\mathcal{P})$ carries a natural poset structure for any $\mathcal{P}$. The quickest way to introduce it is to use the inclusion of the corresponding upper or lower ideals of $\mathcal{P}$. For $\Gamma, \Gamma^{\prime} \in \mathfrak{A} \mathfrak{n}(\mathcal{P})$, we set $\Gamma \leqslant \leqslant_{u p} \Gamma^{\prime}$ if $I(\Gamma) \subset I\left(\Gamma^{\prime}\right)$. The similar use of lower ideals yields the opposite poset structure in $\mathfrak{A} \mathfrak{n}(\mathcal{P})$. That is, letting $\Gamma \leqslant_{l o} \Gamma^{\prime}$ if $I_{-}(\Gamma) \subset I_{-}\left(\Gamma^{\prime}\right)$, we obtain

$$
\Gamma \leqslant{ }_{u p} \Gamma^{\prime} \text { if and only if } \Gamma^{\prime} \leqslant_{l o} \Gamma \text {. }
$$

Sometimes it is convenient to have a separate notation for the (po)sets of upper and lower ideals. Let $\left(\mathcal{J}_{+}(\mathcal{P}), \subseteq\right)\left(\right.$ resp. $\left(\mathcal{J}_{-}(\mathcal{P}), \subseteq\right)$ ) be the poset of upper (resp. lower) ideals in $\mathcal{P}$ with respect to the usual inclusion. The output of the above discussion is that

$$
\begin{aligned}
\left(\mathcal{J}_{+}(\mathcal{P}), \subseteq\right) \simeq\left(\mathfrak{A} \mathfrak{n}(\mathcal{P}), \leqslant_{u p}\right), \quad\left(\mathcal{J}_{-}(\mathcal{P}), \subseteq\right) \simeq\left(\mathfrak{A} \mathfrak{n}(\mathcal{P}), \leqslant_{l o}\right), \\
\quad \text { and }\left(\mathfrak{A} \mathfrak{n}(\mathcal{P}), \leqslant_{l o}\right) \simeq\left(\mathfrak{A} \mathfrak{n}(\mathcal{P}), \leqslant_{u p}\right)^{o p p} .
\end{aligned}
$$

The poset $\left(\mathfrak{A} \mathfrak{n}(\mathcal{P}), \leqslant_{u p}\right)$ is graded, with rank function $\Gamma \mapsto \# I(\Gamma)$. Thus, $\mathcal{M}_{\mathcal{P}}(t)$ is the rank-generating function for $\left(\mathfrak{A} \mathfrak{n}(\mathcal{P}), \leqslant_{u p}\right)$. Note that $\Gamma^{\prime}$ covers $\Gamma$ w.r.t. $\leqslant_{u p}$ if and only if $I\left(\Gamma^{\prime}\right)=I(\Gamma) \cup\{x\}$ for some $x \in \mathcal{P}$; moreover, $I(\Gamma) \cup\{x\}$ is an upper ideal for $x \in \mathcal{P} \backslash I(\Gamma)$ if and only if $x \in \max (\mathcal{P} \backslash I(\Gamma))$. 
2.1. Weight posets and weight multiplicity free representations. Let $\mathfrak{h}$ be a complex reductive algebraic Lie algebra with $r k[\mathfrak{h}, \mathfrak{h}]=m$. Fix a triangular decomposition $\mathfrak{h}=$ $\mathfrak{n}^{-} \oplus \mathfrak{t} \oplus \mathfrak{n}^{+}$. The root system $\Delta_{\mathfrak{h}}=\Delta(\mathfrak{h}, \mathfrak{t})$ is of rank $m$; it is reduced but is not necessarily irreducible. Then $\Delta_{\mathfrak{h}}^{+}$is the set of roots in $\mathfrak{n}^{+}$and $\Pi_{\mathfrak{h}}$ is the set of simple roots in $\Delta_{\mathfrak{h}}^{+}$. Write $\mathcal{X}_{+}$for the set of dominant weights associated with $\Delta_{\mathfrak{h}}^{+}$and $W_{\mathfrak{h}}$ for the Weyl group of $\Delta_{\mathfrak{h}}$.

For a simple $\mathfrak{h}$-module $\mathcal{R}(\lambda)$ with highest weight $\lambda \in \mathcal{X}_{+}$, let $\mathcal{P}(\mathcal{R}(\lambda)$ ) (or merely $\mathcal{P}(\lambda)$ ) be the set of $\mathfrak{t}$-weights in $\mathcal{R}(\lambda)$. Whenever we wish to stress that $\mathcal{P}(\lambda)$ is associated with $\mathfrak{h}$ module, we write $\mathcal{P}(\mathfrak{h}, \lambda)$ for it. The partial order ' $\preccurlyeq$ ' in $\mathcal{P}(\lambda)$ is defined by the requirement that, for $\gamma, \nu \in \mathcal{P}(\lambda), \gamma$ covers $\mu$ if and only if $\gamma-\mu \in \Pi_{\mathfrak{h}}$. Hence $\mu \preccurlyeq \gamma$ if and only if $\gamma-\mu$ is a nonnegative integer linear combination of simple roots. Then $\max \mathcal{P}(\lambda)=\{\lambda\}$. If $\lambda^{*}$ is the highest weight of the dual representation (i.e., $\mathcal{R}(\lambda)^{*}=\mathcal{R}\left(\lambda^{*}\right)$ ), then $-\lambda^{*}$ is the lowest weight of $\mathcal{R}(\lambda)$ and $\min \mathcal{P}(\lambda)=\left\{-\lambda^{*}\right\}$. Let $p$ be any linear function on $\mathfrak{t}^{*}$ such that $p(\alpha)=1$ for all $\alpha \in \Pi_{\mathfrak{h}}$. Then all maximal chains from $-\lambda^{*}$ to $\lambda$ are of length $p\left(\lambda+\lambda^{*}\right)$. Hence $\mathcal{P}(\lambda)$ is a graded poset. For weight posets, it is convenient to assume that the minimal element of $\mathcal{P}(\lambda),-\lambda^{*}$, has rank one. The corresponding rank function $r$ is said to be tuned. It is given by $\mu \in \mathcal{P}(\lambda) \stackrel{r}{\mapsto} p\left(\mu+\lambda^{*}\right)+1$.

If $V$ is any finite-dimensional $\mathfrak{h}$-module, then $\mathcal{P}\left(V^{*}\right)=-\mathcal{P}(V)$, hence the posets $\mathcal{P}\left(V^{*}\right)$ and $\mathcal{P}(V)$ are anti-isomorphic. But we actually have more.

Lemma 2.2. (i) The posets $\mathcal{P}\left(V^{*}\right)$ and $\mathcal{P}(V)$ are naturally isomorphic and (ii) the polynomial $\mathcal{M}_{\mathcal{P}(V)}(t)$ is palindromic, of degree \#P $(V)$.

Proof. (i) Let $w_{0} \in W_{\mathfrak{h}}$ be the longest element. Then $-w_{0}\left(\Pi_{\mathfrak{h}}\right)=\Pi_{\mathfrak{h}}$ and $-w_{0}(\mathcal{P}(V))=$ $\mathcal{P}\left(V^{*}\right)$. Therefore, $\nu \preccurlyeq \mu$ in $\mathcal{P}(V)$ if and only if $-w_{0}(\nu) \preccurlyeq-w_{0}(\mu)$ in $\mathcal{P}\left(V^{*}\right)$.

(ii) Suppose that $I \in \mathcal{J}_{+}(\mathcal{P}(V))$. Since $w_{0}\left(\Delta_{\mathfrak{h}}^{+}\right)=-\Delta_{\mathfrak{h}}^{+}$, we have $w_{0}(I) \in \mathcal{J}_{-}(\mathcal{P}(V))$. Therefore $I^{*}:=\mathcal{P}(V) \backslash w_{0}(I)$ is an upper ideal of complementary cardinality.

Remark 2.3. The reverse operator $\mathfrak{X}_{\mathcal{P}}$ is an element of the symmetric group on $\mathfrak{A} \mathfrak{n}(\mathcal{P})$. Therefore, it can be presented as a product of two involutions. An interesting (perhaps, useful) feature of the weight posets is that $\mathfrak{X}_{\mathcal{P}(V)}$ can be written as such a product in a very explicit way. In Lemma 2.2, we considered, for any $I \in \mathcal{J}_{+}(\mathcal{P}(V))$, the upper ideal $I^{*}=\mathcal{P}(V) \backslash w_{0}(I)$. Then $I^{* *}=I$ and this provides our first ingredient, the involution ' $*^{\prime}$ :

$$
\Gamma=\min (I(\Gamma)) \mapsto \Gamma^{*}=\min \left(I(\Gamma)^{*}\right) .
$$

Using the fact that $w_{0}(\min (I))=\max \left(w_{0}(I)\right)$ for any $I \in \mathcal{J}_{+}(\mathcal{P}(V))$, we obtain

$$
\mathfrak{X}_{\mathcal{P}(V)}(\Gamma)=\max (\mathcal{P}(V) \backslash I(\Gamma))=w_{0}\left(\min \left(\mathcal{P}(V) \backslash w_{0}(I(\Gamma))\right)\right)=w_{0}\left(\Gamma^{*}\right) .
$$

Thus, $\mathfrak{X}_{\mathcal{P}(V)}$ is the product of involutions ' $*^{\prime}$ and $w_{0}$.

We say that $V$ is weight multiplicity free (wmf for short) if every $\mathfrak{t}$-weight space of $V$ is one-dimensional. If $V=\bigoplus_{i} \mathcal{R}\left(\lambda_{i}\right)$ is wmf, then so is each $\mathcal{R}\left(\lambda_{i}\right)$ (but not vice versa!). Then 
$\# \mathcal{P}(V)=\operatorname{dim} V, \mathcal{P}(V)$ is the disjoint union of the posets $\mathcal{P}\left(\lambda_{i}\right)$, and there is a one-to-one correspondence between $\mathcal{J}_{+}(\mathcal{P}(V))$ and the $\left(\mathfrak{t} \oplus \mathfrak{n}^{+}\right)$-stable subspaces of $V$.

The list of the irreducible wmf representations of simple Lie algebras is obtained by R. Howe [10, 4.6], see also [15, Table 1]. Taking tensor products, one derives from it the corresponding list for the semisimple Lie algebras. The following is obvious.

Lemma 2.4. Let $\left(\mathfrak{h}_{i}, \mathcal{R}\left(\lambda_{i}\right)\right), i=1, \ldots, l$, be irreducible representations of reductive Lie algebras. Then

(1) the representation $\mathcal{R}=\mathcal{R}\left(\lambda_{1}\right) \otimes \cdots \otimes \mathcal{R}\left(\lambda_{l}\right)$ of $\mathfrak{h}=\mathfrak{h}_{1} \times \ldots \times \mathfrak{h}_{l}$ is irreducible, with highest weight $\lambda_{1}+\ldots+\lambda_{l}$, and

$$
\mathcal{P}\left(\mathcal{R}\left(\lambda_{1}\right) \otimes \cdots \otimes \mathcal{R}\left(\lambda_{l}\right)\right)=\mathcal{P}\left(\lambda_{1}+\ldots+\lambda_{l}\right)=\mathcal{P}\left(\lambda_{1}\right) \times \ldots \times \mathcal{P}\left(\lambda_{l}\right),
$$

the direct product of the weight posets $\mathcal{P}\left(\lambda_{i}\right)$.

(2) If each $\left(\mathfrak{h}_{i}, \mathcal{R}\left(\lambda_{i}\right)\right)$ is wmf, then so is $(\mathfrak{h}, \mathcal{R})$.

Example 2.5. The root system of $\mathfrak{h}=\mathfrak{s l}_{n}$ is of type $\mathbf{A}_{n-1}$, and we use the common notation for roots, etc., see Tables in $[3,28]$. That is, $\alpha_{i}=\varepsilon_{i}-\varepsilon_{i+1}$ are the simple roots and $\varpi_{i}=$ $\varepsilon_{1}+\ldots+\varepsilon_{i}$ are the fundamental weights, $i=1, \ldots, n-1$. The standard $n$-dimensional representation of $\mathfrak{s l}_{n}$ is wmf and its weights are $\left\{\varepsilon_{i}, i=1, \ldots, n\right\}$. Here $\varepsilon_{1}=\varpi_{1}$ is the highest weight and $\mathcal{P}\left(\varpi_{1}\right)$ is the $n$-element chain $\mathcal{C}_{n}$ :

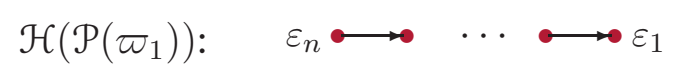

If $\mathfrak{h}=\mathfrak{s l}_{n} \times \mathfrak{s l}_{m}$ and $V=\mathcal{R}\left(\varpi_{1}\right) \otimes \mathcal{R}\left(\varpi_{1}^{\prime}\right)$ is the tensor product of the standard representations, then $\mathcal{P}(V)=\mathcal{P}\left(\varpi_{1}+\varpi_{1}^{\prime}\right) \simeq \mathfrak{C}_{n} \times \mathfrak{C}_{m}$. The Hasse diagram $\mathcal{H}\left(\mathfrak{C}_{n} \times \mathfrak{C}_{m}\right)$ is the rectangle of size $m \times n$. Clearly, all posets $\mathfrak{C}_{n_{1}} \times \cdots \times \mathcal{C}_{n_{l}}$ are associated with wmf representations, but one shouldn't expect much from them, if $l \geqslant 4$, see Section 5 .

For a graded poset $\mathcal{P}$, let $\mathcal{P}_{i}$ denote the set of elements of rank $i$. The sets $\mathcal{P}_{i}$ are said to be the (rank) levels of $\mathcal{P}$. Suppose that $\mathcal{P}=\bigsqcup_{i=1}^{d} \mathcal{P}_{i}$. Then $\mathcal{P}$ is rank symmetric if $\# \mathcal{P}_{i}=\# \mathcal{P}_{d+1-i}$ for all $i$; it is rank unimodal if $\# \mathcal{P}_{1} \leqslant \# \mathcal{P}_{2} \leqslant \cdots \leqslant \# \mathcal{P}_{k} \geqslant \# \mathcal{P}_{k+1} \geqslant \cdots \geqslant \# \mathcal{P}_{d}$ for some $1 \leqslant k \leqslant d$. The poset $\mathcal{P}$ is said to be Sperner, if the largest size of an antichain is equal to $\max \left\{\# \mathcal{P}_{i}, 1 \leqslant i \leqslant d\right\}$.

Lemma 2.6. For any simple wmf $\mathfrak{h}$-module $\mathcal{R}(\lambda)$, the graded poset $\mathcal{P}(\lambda)$ is rank symmetric, rank unimodal, and Sperner.

Proof. Consider a principal $\mathfrak{s l}_{2}$-subalgebra $\mathfrak{a} \subset[\mathfrak{h}, \mathfrak{h}]$ associated with our fixed triangular decomposition of $\mathfrak{h}$. That is, $\mathfrak{a} \simeq \mathfrak{s l}_{2}$ has a basis $(X, H, Y)$ such that $[X, Y]=H,[H, X]=$ $2 X,[H, Y]=-2 Y, H \in \mathfrak{t}$ is a regular element, and $X$ (resp. $Y$ ) is a sum of root vectors corresponding to $\Pi_{\mathfrak{h}}\left(\right.$ resp. $\left.-\Pi_{\mathfrak{h}}\right)$. Then the representation of $\mathfrak{a}$ on $\mathcal{R}(\lambda)$ translates into a representation of $\mathfrak{a}$ on the graded poset $\mathcal{P}(\lambda)$, as defined in [19]. Therefore, the main result of [19] yields all the assertions. 
It follows from [19] that the poset $\mathcal{P}(\lambda)$ is also strongly Sperner, but we do need this here.

Remark 2.7. The idea to use $\mathfrak{s l}_{2}$-subalgebras for obtaining properties of irreducible representations goes back to E.B. Dynkin. In 1950, he invented principal $\mathfrak{s l}_{2}$-subalgebras of semisimple Lie algebras and proved, using them, that a certain partition of $\mathcal{R}(\lambda)$ into layers, $\mathcal{R}(\lambda)=\bigoplus_{j=1}^{N} \mathcal{R}(\lambda)_{j}$, yields a symmetric unimodal sequence $\left\{d_{j}=\operatorname{dim} \mathcal{R}(\lambda)_{j}\right\}_{j=1}^{N}$, see [8, Theorem 4]. In the special case of wmf representations, one has $d_{j}=\# \mathcal{P}(\lambda)_{j}$ and Dynkin's result translates into the assertion that $\mathcal{P}(\lambda)$ is rank symmetric and rank unimodal.

2.2. Gradings of simple Lie algebras. Interesting wmf representations and weight posets are associated with gradings of simple Lie algebras. We refer to [28, Ch. 3, $\S 3]$ for generalities on gradings. In what follows, $\mathfrak{g}$ is a complex simple Lie algebra with a fixed triangular decomposition $\mathfrak{g}=\mathfrak{u}^{-} \oplus \mathfrak{t} \oplus \mathfrak{u}^{+}$. The associated root system is $\Delta=\Delta(\mathfrak{g}, \mathfrak{t}) \subset \mathfrak{t}^{*}$, and we use all the relevant notation on $\Delta$ presented in Introduction. Additionally, $\mathfrak{t}_{\mathbb{R}}^{*}$ is the $\mathbb{R}$-span of $\Delta$ in $\mathfrak{t}^{*}$ and $($,$) is a W$-invariant scalar product in $\mathfrak{t}_{\mathbb{R}}^{*}$. For $\gamma \in \Delta$, we set $\gamma^{\vee}=2 \gamma /(\gamma, \gamma)$. Then $\Delta^{\vee}=\left\{\gamma^{\vee} \mid \gamma \in \Delta\right\}$ is the dual root system, $\left(\Delta^{\vee}\right)^{+}=\left(\Delta^{+}\right)^{\vee}$ is a set of positive roots in $\Delta^{\vee}$, and $\Pi^{\vee}=\left\{\alpha^{\vee} \mid \alpha \in \Pi\right\}$ is the set of simple roots in $\left(\Delta^{\vee}\right)^{+}$. However, $\theta^{\vee}$ appears to be the highest root in $\left(\Delta^{\vee}\right)^{+}$is and only if all roots of $\Delta$ have the same length (i.e., $\Delta \in\{\mathrm{ADE}\})$. Write $s_{\gamma}$ for the reflection in $W$ with respect to $\gamma \in \Delta$. Note that $s_{\gamma}=s_{\gamma^{\vee}}$.

- Let $\mathfrak{g}=\bigoplus_{i \in \mathbb{Z}} \mathfrak{g}(i)$ be a $\mathbb{Z}$-grading. Since any derivation of $\mathfrak{g}$ is inner, $\mathfrak{g}(i)=\{x \in$ $\mathfrak{g} \mid[\tilde{h}, x]=i x\}$ for some $\tilde{h} \in \mathfrak{g}(0)$. The element $\tilde{h}$ is said to be defining for the grading in question. Since $\tilde{h}$ is semisimple, $\mathfrak{g}(0)$ is reductive, $r k \mathfrak{g}=r \mathbf{k} \mathfrak{g}(0)$, and $\mathfrak{g}(i)$ is a wmf $\mathfrak{g}(0)$ module for $i \neq 0$. Because $\tilde{h}$ lies in the centre of $\mathfrak{g}(0)$, it is convenient to introduce a reductive subalgebra $\widetilde{\mathfrak{g}(0)}$ such that $\mathfrak{g}(0)=\widetilde{\mathfrak{g}(0)} \oplus\langle\tilde{h}\rangle$.

Without loss of generality, one may assume that $\tilde{h} \in \mathfrak{t}$ and $\alpha(\tilde{h}) \geqslant 0$ for all $\alpha \in \Pi$. Then $\mathfrak{t} \subset \mathfrak{g}(0)$ and the grading is fully determined by the partition $\Pi=\bigsqcup_{i \geqslant 0} \Pi(i)$, where $\Pi(i)=\{\alpha \in \Pi \mid \alpha(\tilde{h})=i\}$. If $\Delta(i)=\{\gamma \in \Delta \mid \gamma(\tilde{h})=i\}$, then $\Delta(i)$ is the set of roots of $\mathfrak{g}(i)$, and $\Delta=\bigsqcup_{i \in \mathbb{Z}} \Delta(i)$. We repeatedly use the property that the last partition is a "grading" of $\Delta$, i.e., if $\gamma \in \Delta(i), \gamma^{\prime} \in \Delta(j)$, and $\gamma+\gamma^{\prime}$ is a root, then $\gamma+\gamma^{\prime} \in \Delta(i+j)$.

In this setting, we automatically obtain a distinguished triangular decomposition:

$$
\mathfrak{g}(0)=\left(\mathfrak{g}(0) \cap \mathfrak{u}^{-}\right) \oplus \mathfrak{t} \oplus\left(\mathfrak{g}(0) \cap \mathfrak{u}^{+}\right) .
$$

Then $\Delta(0)^{+}:=\Delta^{+} \cap \Delta(0)$ is the set of roots of $\mathfrak{g}(0) \cap \mathfrak{u}^{+}, \Pi(0)$ is the set of simple roots in $\Delta(0)^{+}$, and

$$
\Delta^{+}=\Delta(0)^{+} \sqcup \Delta(1) \sqcup \Delta(2) \sqcup \ldots
$$

We may (and will) use the notation and results of Section 2.1 with $\mathfrak{h}=\mathfrak{g}(0), \mathfrak{n}^{+}=\mathfrak{g}(0) \cap \mathfrak{u}^{+}$, $\Pi_{\mathfrak{h}}=\Pi(0)$, etc. For instance, $\Delta(i)=\mathcal{P}(\mathfrak{g}(i))$. The Weyl group of $\Delta(0), W(0)$, is a parabolic subgroup of $W$. Let $W^{0}$ denote the set of representatives of minimal length for the cosets 
$W / W(0)$. In other words [11, 1.10],

$$
W^{0}=\left\{w \in W \mid w(\alpha) \in \Delta^{+} \quad \forall \alpha \in \Delta(0)^{+}\right\} .
$$

Remark 2.8. Since $\mathfrak{g}(i)$ and $\mathfrak{g}(-i)$ are dual $\mathfrak{g}(0)$-modules, the posets $\Delta(i)$ and $\Delta(-i)$ are isomorphic. Furthermore, if one is interested in possible simple $\mathfrak{g}(0)$-modules occurring in $\mathfrak{g}(i)$ with $i>0$, then it is enough to consider only the simple $\mathfrak{g}(0)$-modules in $\mathfrak{g}(1)$, see [27, $\S 1.2, \S 2.1]$. (For $i>1$, the problem is reduced to considering the induced grading of a certain simple subalgebra of $\mathfrak{g}$.) For this reason, it suffices to consider defining elements $\tilde{h} \in \mathfrak{t}$ such that $\alpha(\tilde{h}) \in\{0,1\}$, i.e., $\Pi=\Pi(0) \sqcup \Pi(1)$. The corresponding $\mathbb{Z}$-gradings are said to be standard. Moreover, if $\# \Pi(1)=k$, then we call it a $k$-standard grading. A standard $\mathbb{Z}$-grading can be represented by the Dynkin diagram of $\mathfrak{g}$, where the vertices in $\Pi(1)$ are marked. If $\Pi(1)=\left\{\alpha_{i_{1}}, \ldots, \alpha_{i_{k}}\right\}$, then the $\alpha_{i_{j}}$ 's are precisely the lowest weights of the simple $\mathfrak{g}(0)$-modules in $\mathfrak{g}(1)$, the centre of $\mathfrak{g}(0)$ is $k$-dimensional, and $\mathfrak{g}(1)$ is a direct sum of $k$ simple $\mathfrak{g}(0)$-modules. Thus, for the standard $\mathbb{Z}$-gradings, one obtains

$\widetilde{\mathfrak{g}(0)}$ is semisimple $\Leftrightarrow$ the grading is 1-standard $\Leftrightarrow \mathfrak{g}(1)$ is a simple $\widetilde{\mathfrak{g}(0)}$-module.

Remark 2.9. The weight posets $\Delta(i), i>0$, can be visualised as follows. If $\gamma^{\prime}-\gamma=\alpha \in \Pi$, then the edge connecting $\gamma$ and $\gamma^{\prime}$ in the Hasse diagram $\mathcal{H}\left(\Delta^{+}\right)$is said to be of type $\alpha$. Given a standard $\mathbb{Z}$-grading of $\mathfrak{g}$, let us remove from $\mathcal{H}\left(\Delta^{+}\right)$all the edges of types in $\Pi(1)$. This yields a disconnected graph. Each connected component of it is the Hasse diagram of either the set of positive roots of a simple factor of $\mathfrak{g}(0)$ (if it contains roots from $\Pi(0)$ ) or the weight poset of a simple $\mathfrak{g}(0)$-module in some $\mathfrak{g}(i), i>0$. The set of weights of a simple $\mathfrak{g}(0)$-module in some $\mathfrak{g}(i), i \geqslant 1$, is precisely the set of roots $\gamma$ with fixed values $[\gamma: \alpha]$ for all $\alpha \in \Pi(1)$, see e.g. [28, 3.5]. Therefore,

- each weight poset $\Delta(i), i \geqslant 1$, is a subposet of $\Delta^{+}$.

- the connected component containing a node $\alpha \in \Pi(1)$ is the Hasse diagram of the weight poset of a simple $\mathfrak{g}(0)$-module in $\mathfrak{g}(1)$,

- the tuned rank function for the whole poset $\Delta(1)$ is the restriction to $\Delta(1)$ of the usual height function ht: $\Delta^{+} \rightarrow \mathbb{N}$.

- Let $\mathfrak{g}=\bigoplus_{i \in \mathbb{Z}_{m}} \mathfrak{g}_{i}$ be a periodic (or $\mathbb{Z}_{m^{-}}$) grading. Such gradings are in a one-to-one correspondence with the automorphisms $\sigma \in \operatorname{Aut}(\mathfrak{g})$ of order $m$. Here again $\mathfrak{g}_{0}$ is reductive and $\mathfrak{g}_{i}$ is a $\mathfrak{g}_{0}$-module. If $\sigma$ is inner, then $r k \mathfrak{g}_{0}=r k \mathfrak{g}$ and $\mathfrak{g}_{i}$ is a wmf $\mathfrak{g}_{0}$-module for $i \neq 0$. For inner automorphisms $\sigma$, one may further assume that $\mathfrak{t} \subset \mathfrak{g}_{0}$ and obtain the partition $\Delta=\bigsqcup_{i=0}^{m-1} \Delta_{i}$ with $\Delta_{i}=\mathcal{P}\left(\mathfrak{g}_{i}\right)$. A classification of periodic automorphisms of $\mathfrak{g}$ is obtained by V.G. Kac (1969). In particular, an explicit description of inner periodic automorphisms of $\mathfrak{g}$ and the respective $\mathfrak{g}_{0}$-modules $\mathfrak{g}_{1}$ can be given in terms of the extended Dynkin diagram of $\mathfrak{g}$, see $[27, \S 8]$ or $[28, \mathrm{Ch} .3, \S 3.7]$ for a thorough self-contained description. 
Likewise, for periodic gradings, it suffices to consider $\mathfrak{g}_{0}$-modules $\mathfrak{g}_{1}$. We mention the following useful property of periodic gradings: $\mathfrak{g}_{1}$ is a simple $\mathfrak{g}_{0}$-module if and only if $\mathfrak{g}_{0}$ is semisimple, see [27, Prop.18]. Note, however, that all $\Delta_{i}$ are not usually subsets of $\Delta^{+}$! Therefore, the rank function for $\Delta_{1}$ has no relation to ht ( ).

Our main interest lies in the study of representations (posets) related to $\mathbb{Z}$-gradings. In Section 5, we use wmf representations associated with periodic gradings to demonstrate that properties of the antichains and reverse operators become worse for them.

Remark 2.10. In this article, we are primarily interested in combinatorial properties of weight posets. It is therefore helpful to keep in mind that the weight posets of two wmf representations of different Lie algebras can be isomorphic. A list of such isomorphisms appears in [15, Theorem 2.1].

2.3. Special classes of $\mathbb{Z}$-gradings. Our goal is to demonstrate that the weight posets of the form $\Delta(1)$ for $\mathbb{Z}$-gradings of $\mathfrak{g}$ exhibit the best possible properties related to antichains, their $t$-analogues, and reverse operators. We will consider in details the following two cases:

The abelian gradings: $\mathfrak{g}=\mathfrak{g}(-1) \oplus \mathfrak{g}(0) \oplus \mathfrak{g}(1)$.

Here $\mathfrak{g}(0) \bigoplus \mathfrak{g}(1)$ is a parabolic subalgebra and $\mathfrak{g}(1)$ is its (abelian!) nilradical. In this case $\mathfrak{g}(1)$ is a simple $\mathfrak{g}(0)$-module and therefore $\widetilde{\mathfrak{g}(0)}$ is semisimple and $\Pi(1)=\left\{\alpha_{i}\right\}$. The admissible simple roots $\alpha_{i}$ are characterised by the property that $\left[\theta: \alpha_{i}\right]=1$. Therefore, such gradings exist if and only if $\mathfrak{g} \neq \mathbf{G}_{2}, \mathbf{F}_{4}, \mathbf{E}_{8}$. The following table provides the admissible simple roots (with numbering as in [28, Table 1]):

\begin{tabular}{|cc||cc|}
\hline $\mathbf{A}_{n}$ & $\alpha_{1}, \ldots, \alpha_{n}$ & $\mathbf{D}_{n}, n \geqslant 4$ & $\alpha_{1}, \alpha_{n-1}, \alpha_{n}$ \\
\hline $\mathbf{B}_{n}, n \geqslant 2$ & $\alpha_{1}$ & $\mathbf{E}_{6}$ & $\alpha_{1}, \alpha_{5}$ \\
\hline $\mathbf{C}_{n}, n \geqslant 2$ & $\alpha_{n}$ & $\mathbf{E}_{7}$ & $\alpha_{1}$ \\
\hline
\end{tabular}

In particular, all 1-standard $\mathbb{Z}$-gradings for $\mathbf{A}_{n}$ are abelian! If an abelian grading corresponds to $\alpha_{i} \in \Pi$, then upon the identification of $\mathfrak{t}_{\mathbb{R}}$ and $\mathfrak{t}_{\mathbb{R}}^{*}$, the defining element $\tilde{h}$ appears to be the minuscule fundamental weight $\varphi_{i}^{\vee}$ of the dual root system $\Delta^{\vee}$. Indeed, the weight $\varphi_{i}^{\vee}$ satisfies the relation

$$
\left(\varphi_{i}^{\vee},\left(\gamma^{\vee}\right)^{\vee}\right)=\left(\varphi_{i}^{\vee}, \gamma\right)=\gamma(\tilde{h}) \in\{-1,0,1\} \quad \text { for all } \gamma^{\vee} \in \Delta^{\vee} .
$$

Hence it is minuscule [4, Ch. VIII, $\left.\S 7, \mathrm{n}^{0} 3\right]$. Furthermore, $\left(\varphi_{i}^{\vee}, \alpha_{i}\right)=1$ and $\left(\varphi_{i}^{\vee}, \alpha\right)=0$ for $\alpha \in \Pi \backslash\left\{\alpha_{i}\right\}$. Hence $\varphi_{i}^{\vee}$ is fundamental. Note also that $W(0)$ is the stabiliser of $\varphi_{i}^{\vee}$ in $W$.

The extra-special gradings: $\mathfrak{g}=\mathfrak{g}(-2) \bigoplus \mathfrak{g}(-1) \oplus \mathfrak{g}(0) \bigoplus \mathfrak{g}(1) \bigoplus \mathfrak{g}(2) \& \operatorname{dim} \mathfrak{g}(2)=1$. Any simple Lie algebra has a unique, up to conjugation, $\mathbb{Z}$-grading of this form, and w.l.o.g. we may assume that $\Delta(2)=\{\theta\}$. Upon the identification of $\mathfrak{t}_{\mathbb{R}}$ and $\mathfrak{t}_{\mathbb{R}}^{*}$, the defining element $\tilde{h}$ is recognised as the coroot $\theta^{\vee}$. That is, $\Delta(i)=\left\{\gamma \in \Delta \mid\left(\gamma, \theta^{\vee}\right)=i\right\}$ and 
$W(0)$ is the stabiliser of $\theta$ ( or $\theta^{\vee}$ ) in $W$. For $\mathbf{A}_{1}$ only, we have $\Delta(1)=\varnothing$, i.e., the grading appears to be not standard. (This case better fits in the setting of abelian gradings.) For all other simple Lie algebras, $\theta \notin \Pi$ and the extra-special grading is standard. Then $\Pi(1)=\left\{\alpha \in \Pi \mid\left(\gamma, \theta^{\vee}\right) \neq 0\right\}$. Therefore $\widetilde{\mathfrak{g}(0)}$ is semisimple and $\mathfrak{g}(1)$ is a simple $\mathfrak{g}(0)$ module if and only if $\theta$ is a multiple of a fundamental weight, i.e., $\mathfrak{g}$ is not of type $\mathbf{A}_{n}$.

Note also that $\mathfrak{g}(1)$ is a symplectic $\widetilde{\mathfrak{g}(0)}$-module (hence $\operatorname{dim} \mathfrak{g}(1)$ is even) and $\mathfrak{g}(1) \bigoplus \mathfrak{g}(2)$ is a Heisenberg Lie algebra.

\section{ANTICHAINS AND UPPER IDEALS IN THE ABELIAN CASE}

In this section, only abelian $\mathbb{Z}$-gradings of $\mathfrak{g}$ are considered. We give a description of the antichains and upper ideals in the poset $\Delta(1)$ and compute the corresponding $\mathcal{M}$ - and $\mathcal{N}$-polynomials.

For $w \in W$, let $N(w) \subset \Delta^{+}$be the inversion set and $\ell(w)$ the length of $w$. Recall that $\ell(w)=\# N(w)$. It is readily seen that both $N(w)$ and $\Delta^{+} \backslash N(w)$ are closed subsets of $\Delta^{+}$. (That is, if $\gamma_{1}, \gamma_{2} \in N(w)$ and $\gamma_{1}+\gamma_{2}$ is a root, then $\gamma_{1}+\gamma_{2} \in N(w)$; and likewise for $\Delta^{+} \backslash N(w)$.) Conversely, if $M \subset \Delta^{+}$has the property that both $M$ and $\Delta^{+} \backslash M$ are closed, then $M=N(w)$ for a unique $w \in W$, see e.g. [18, p. 663].

Theorem 3.1. In the abelian case, there is a natural bijection $W^{0} \stackrel{1: 1}{\longleftrightarrow} \mathfrak{A} \mathfrak{n}(\Delta(1))$ that takes $w \in W^{0}$ to $\Gamma_{w}:=\min (\Delta(1) \backslash N(w))$. Furthermore,

(i) $I_{w}=\Delta(1) \backslash N(w)$ is an upper ideal in $\Delta(1)$;

(ii) if $\gamma \in I_{w}$, then $\gamma \in \min \left(I_{w}\right)$ if and only if $w(\gamma) \in \Pi$; in other words, $\Gamma_{w}=\min \left(I_{w}\right)=$ $w^{-1}(\Pi) \cap \Delta(1)$;

(iii) if $\gamma \in N(w)$, then $\gamma \in \max (N(w))$ if and only if $w(\gamma) \in-\Pi$; in other words, $\max (N(w))=\max \left(\Delta(1) \backslash I_{w}\right)=-w^{-1}(\Pi) \cap \Delta(1)$.

In particular, $\# \mathfrak{A} \mathfrak{n}(\Delta(1))=\#(W / W(0))$ and $\# I_{w}=\operatorname{dim} \mathfrak{g}(1)-\ell(w)$.

Proof. We have $\Delta^{+}=\Delta(0)^{+} \cup \Delta(1)$. If $w \in W^{0}$, then $N(w) \subset \Delta(1)$ and we set

$$
I_{w}:=\Delta(1) \backslash N(w)=\{\gamma \in \Delta(1) \mid w(\gamma)>0\} .
$$

1) If $\delta \in \Delta(0)^{+}, \gamma \in I_{w}$, and $\delta+\gamma$ is a root, then $\delta+\gamma \in I_{w}$, since $\Delta^{+} \backslash N(w)$ is closed. Hence $I_{w}$ is an upper ideal of the poset $\Delta(1)$. Conversely, it is immediate that if $I \subset \Delta(1)$ is an upper ideal, then both $\Delta(1) \backslash I$ and $I \cup \Delta(0)^{+}$are closed. Hence $\Delta(1) \backslash I=N(w)$ for a unique $w \in W^{0}$. This yields the desired bijection and (i).

2) Suppose that $\gamma \in I_{w}$ and $\gamma \notin \min \left(I_{w}\right)$. Then $\gamma=\delta+\gamma^{\prime}$ for some $\delta \in \Pi(0)$ and $\gamma^{\prime} \in I_{w}$. Hence $w(\gamma)=w(\delta)+w\left(\gamma^{\prime}\right)$ is a sum of positive roots, i.e., $w(\gamma) \notin \Pi$.

Conversely, assume that $w(\gamma) \notin \Pi$, i.e., $w(\gamma)=\mu_{1}+\mu_{2}$ with $\mu_{i} \in \Delta^{+}$. Then

$$
w^{-1}\left(\mu_{1}\right)+w^{-1}\left(\mu_{2}\right)=\gamma \in \Delta(1) .
$$


We may assume that $w^{-1}\left(\mu_{1}\right) \in \Delta(0)$ and $w^{-1}\left(\mu_{2}\right) \in \Delta(1)$. Then $w^{-1}\left(\mu_{2}\right) \in I_{w}$. The root $w^{-1}\left(\mu_{1}\right)$ is either positive or negative. The assumption $w^{-1}\left(\mu_{1}\right) \in-\Delta(0)^{+}$contradicts the fact that $w \in W^{0}$, and if $w^{-1}\left(\mu_{1}\right) \in \Delta(0)^{+}$, then $\gamma-w^{-1}\left(\mu_{1}\right) \in I_{w}$, hence $\gamma \notin \min \left(I_{w}\right)$. This proves (ii).

3) The proof of (iii) is similar to the previous argument and left to the reader.

Corollary 3.2. For $w \in W^{0}$, we have $\# \max (N(w))+\# \Gamma_{w} \leqslant \mathrm{rkg}$.

Proof. We have $w\left(\Gamma_{w}\right) \subset \Pi, w(-\max (N(w))) \subset \Pi$, and all these roots are different.

Remark. Since $\Gamma_{w}$ is an antichain and $\Delta(2)=\varnothing$, the roots in $\Gamma_{w}$ are pairwise strongly orthogonal. That is, $\gamma \pm \gamma^{\prime}$ is not a root for all $\gamma, \gamma^{\prime} \in \Gamma_{w}$. Therefore, $w\left(\Gamma_{w}\right)$ is a strongly orthogonal set of simple roots, i.e., $w\left(\Gamma_{w}\right)$ represents a totally disjoint subset of the Dynkin diagram. The same also holds for $\max (N(w))$ and $w(-\max (N(w))) \subset \Pi$. Hence $w\left(\Gamma_{w}\right)$ and $w(-\max (N(w)))$ are totally disjoint subsets of $\Pi$ without common elements. One can verify that the equality

$$
\# \Gamma_{w}+\# \max (N(w))=\mathbf{r k g} \mathfrak{g} \text {, i.e., } w\left(\Gamma_{w}\right) \bigsqcup w(-\max (N(w)))=\Pi
$$

occurs for some $w \in W^{0}$ if and only if $\Delta$ is of type $\mathbf{A}_{n}$ or $\mathbf{C}_{n}$. (And since the Dynkin diagram is a tree, such a partition of $\Pi$ into two totally disjoint subsets is unique, up to permutation). For $\mathbf{C}_{n}$, one considers the unique abelian $\mathbb{Z}$-grading with $\Delta(1)=\left\{\varepsilon_{i}+\varepsilon_{j} \mid\right.$ $1 \leqslant i \leqslant j \leqslant n\}$. Then one of the possibilities is $\Gamma_{w}=\left\{\varepsilon_{1}+\varepsilon_{n}, \varepsilon_{2}+\varepsilon_{n-1}, \ldots\right\}$ and $\max (N(w))=\left\{\varepsilon_{2}+\varepsilon_{n}, \varepsilon_{3}+\varepsilon_{n-1}, \ldots\right\}$. Here $\# \Gamma_{w}=\left[\frac{n+1}{2}\right]$ and $\# \max (N(w))=\left[\frac{n}{2}\right]$. For $\mathbf{A}_{n}$, one has to take the abelian grading corresponding to $\alpha_{i}$ with $i=\left[\frac{n+1}{2}\right]$ or $i=n+1-\left[\frac{n+1}{2}\right]$. (Hence there are two possible gradings for $\mathbf{A}_{2 k}$ and only one grading for $\mathbf{A}_{2 k-1}$.) The details are left to the reader.

For any subset $S \subset W$, we define its Poincaré polynomial by $\boldsymbol{S}(t)=\sum_{w \in S} t^{\ell(w)}$.

Theorem 3.3. For the abelian gradings, we have $\mathcal{M}_{\Delta(1)}(t)=\prod_{\gamma \in \Delta(1)} \frac{1-t^{\mathrm{ht}(\gamma)+1}}{1-t^{\mathrm{ht}(\gamma)}}$.

Proof. By the Kostant-Macdonald identity [12, Cor. 2.5], we have

$$
\boldsymbol{W}(t)=\prod_{\gamma \in \Delta^{+}} \frac{1-t^{\mathrm{ht}(\gamma)+1}}{1-t^{\mathrm{ht}(\gamma)}} \text { and } \boldsymbol{W}(\mathbf{0})(t)=\prod_{\gamma \in \Delta(0)^{+}} \frac{1-t^{\mathrm{ht}(\gamma)+1}}{1-t^{\mathrm{ht}(\gamma)}}
$$

The bijection $W^{0} \times W(0) \stackrel{\sim}{\longrightarrow} W$ has the property that if $w_{1} \in W^{0}$ and $w_{2} \in W(0)$, then $\ell\left(w_{1} w_{2}\right)=\ell\left(w_{1}\right)+\ell\left(w_{2}\right)$, see $[11,1.10]$. It follows that $\prod_{\gamma \in \Delta(1)} \frac{1-t^{\mathrm{ht}(\gamma)+1}}{1-t^{\mathrm{ht}(\gamma)}}=\boldsymbol{W}^{\mathbf{0}}(t)$ and

$$
\left[t^{i}\right] \prod_{\gamma \in \Delta(1)} \frac{1-t^{\mathrm{ht}(\gamma)+1}}{1-t^{\mathrm{ht}(\gamma)}}=\#\left\{w \in W^{0} \mid \ell(w)=i\right\} .
$$


By Theorem 3.1, the latter equals the number of upper ideals of cardinality $\# \Delta(1)-i$. Since $\mathcal{M}_{\Delta(1)}(t)$ is palindromic and of degree $\# \Delta(1)$ (Lemma 2.2), we are done.

Corollary 3.4. For the abelian gradings, we have $\# \mathfrak{A} \mathfrak{n}(\Delta(1))=\mathcal{M}_{\Delta(1)}(1)=\prod_{\gamma \in \Delta(1)} \frac{\mathrm{ht}(\gamma)+1}{\mathrm{ht}(\gamma)}$.

We are not aware of a uniform general expression for the $\mathcal{N}$-polynomials related to the abelian case, but it is not hard to compute them directly. The resulting list is provided below. For each item, we point out the corresponding simple root $\alpha_{i}$ of $\mathfrak{g}$, the semisimple Lie algebra $\widetilde{\mathfrak{g}(0)}$, and the highest weight $\psi$ of the irreducible representation of $\widetilde{\mathfrak{g}(0)}$ in $\mathfrak{g}(1)$. Write $\varpi_{i}$ for the $i$-th fundamental weight of a simple factor of $\widetilde{\mathfrak{g}(0)}$.

The abelian $\mathbb{Z}$-gradings: $\left(\mathfrak{g}=\bigoplus_{j=-1}^{1} \mathfrak{g}(j), \alpha_{i}\right) \sim(\widetilde{\mathfrak{g}(0)}, \psi)$ and $\mathcal{N}$-polynomials:

1. $\left(\mathbf{A}_{n+m-1}, \alpha_{m}\right) \sim\left(\mathbf{A}_{n-1} \times \mathbf{A}_{m-1}, \varpi_{1}+\varpi_{1}^{\prime}\right), \operatorname{dim} \mathcal{R}\left(\varpi_{1}+\varpi_{1}^{\prime}\right)=n m, \# \mathfrak{A} \mathfrak{n}\left(\mathcal{P}\left(\varpi_{1}+\varpi_{1}^{\prime}\right)\right)=\left(\begin{array}{c}n+m \\ m\end{array}\right)$. $\mathcal{N}_{\Delta(1)}(t)=\sum_{i \geqslant 0}\left(\begin{array}{c}n \\ i\end{array}\right)\left(\begin{array}{c}m \\ i\end{array}\right) t^{i}, \mathcal{N}_{\Delta(1)}^{\prime}(1)=\frac{(n+m-1) !}{(n-1) !(m-1) !}, \mathcal{N}_{\Delta(1)}^{\prime}(1) / \mathcal{N}_{\Delta(1)}(1)=\frac{n \cdot m}{n+m}$

2. $\left(\mathbf{B}_{n}, \alpha_{1}\right) \leadsto\left(\mathbf{B}_{n-1}, \varpi_{1}\right), \quad \operatorname{dim} \mathcal{R}\left(\varpi_{1}\right)=2 n-1, \quad \# \mathfrak{A} \mathfrak{n}\left(\mathcal{P}\left(\varpi_{1}\right)\right)=2 n$.

$$
\mathcal{N}_{\Delta(1)}(t)=1+(2 n-1) t, \quad \mathcal{N}_{\Delta(1)}^{\prime}(1) / \mathcal{N}_{\Delta(1)}(1)=\frac{2 n-1}{2 n} .
$$

3. $\left(\mathbf{C}_{n}, \alpha_{n}\right) \sim\left(\mathbf{A}_{n-1}, 2 \varpi_{1}\right), \quad \operatorname{dim} \mathcal{R}\left(2 \varpi_{1}\right)=n(n+1) / 2, \# \mathfrak{A n}\left(\mathcal{P}\left(2 \varpi_{1}\right)\right)=2^{n}$.

$$
\mathcal{N}_{\Delta(1)}(t)=\sum_{i \geqslant 0}\left(\begin{array}{c}
n+1 \\
2 i
\end{array}\right) t^{i}, \quad \mathcal{N}_{\Delta(1)}^{\prime}(1) / \mathcal{N}_{\Delta(1)}(1)=\frac{n+1}{4} .
$$

4. $\left(\mathbf{D}_{n}, \alpha_{n-1}\right.$ or $\left.\alpha_{n}\right) \leadsto\left(\mathbf{A}_{n-1}, \varpi_{2}\right), \quad \operatorname{dim} \mathcal{R}\left(\varpi_{2}\right)=n(n-1) / 2, \# \mathfrak{A} \mathfrak{n}\left(\mathcal{P}\left(\varpi_{2}\right)\right)=2^{n-1}$.

$$
\mathcal{N}_{\Delta(1)}(t)=\sum_{i \geqslant 0}\left(\begin{array}{c}
n \\
2 i
\end{array}\right) t^{i}, \quad \mathcal{N}_{\Delta(1)}^{\prime}(1) / \mathcal{N}_{\Delta(1)}(1)=\frac{n}{4} .
$$

5. $\left(\mathbf{D}_{n}, \alpha_{1}\right) \leadsto\left(\mathbf{D}_{n-1}, \varpi_{1}\right), \quad \operatorname{dim} \mathcal{R}\left(\varpi_{1}\right)=2 n-2, \quad \# \mathfrak{A} \mathfrak{n}\left(\mathcal{P}\left(\varpi_{1}\right)\right)=2 n$.

$$
\mathcal{N}_{\Delta(1)}(t)=1+(2 n-4) t+t^{2}, \quad \mathcal{N}_{\Delta(1)}^{\prime}(1) / \mathcal{N}_{\Delta(1)}(1)=1 \text {. }
$$

6. $\left(\mathbf{E}_{6}, \alpha_{1}\right.$ or $\left.\alpha_{5}\right) \leadsto\left(\mathbf{D}_{5}, \varpi_{4}\right.$ or $\left.\varpi_{5}\right), \quad \operatorname{dim} \mathcal{R}\left(\varpi_{5}\right)=16, \quad \# \mathfrak{A} \mathfrak{n}\left(\mathcal{P}\left(\varpi_{5}\right)\right)=\frac{9 \cdot 12}{1 \cdot 4}=27$.

$$
\mathcal{N}_{\Delta(1)}(t)=1+16 t+10 t^{2}, \quad \mathcal{N}_{\Delta(1)}^{\prime}(1) / \mathcal{N}_{\Delta(1)}(1)=16 / 12 \text {. }
$$

7. $\left(\mathbf{E}_{7}, \alpha_{1}\right) \leadsto\left(\mathbf{E}_{6}, \varpi_{1}\right), \quad \operatorname{dim} \mathcal{R}\left(\varpi_{1}\right)=27, \quad \# \mathfrak{A} \mathfrak{n}\left(\mathcal{P}\left(\varpi_{1}\right)\right)=\frac{10 \cdot 14 \cdot 18}{1 \cdot 5 \cdot 9}=56$.

$$
\mathcal{N}_{\Delta(1)}(t)=1+27 t+27 t^{2}+t^{3}, \quad \mathcal{N}_{\Delta(1)}^{\prime}(1) / \mathcal{N}_{\Delta(1)}(1)=27 / 18
$$

\section{Comments on computations:}

- For item $1, \Delta(1)$ is the direct product of two chains, see Example 2.5. Here $\mathcal{H}(\Delta(1))=$ $\mathcal{H}\left(\mathcal{P}\left(\varpi_{1}+\varpi_{1}^{\prime}\right)\right)$ is a rectangle and computations are straightforward.

- For item $2, \Delta(1)=\mathcal{P}\left(\mathbf{B}_{n-1}, \varpi_{1}\right) \simeq \mathfrak{C}_{2 n-1}$;

- For item $3, \mathfrak{g}(1)$ is the unique maximal abelian $\mathfrak{b}$-ideal in $\mathfrak{u}$, and $\mathcal{N}_{\Delta(1)}(t)$ is the upper covering polynomial for the poset of all abelian ideals, see [16, Theorem 6.2].

- Item 4 is related to item 3 via a shift of rank of $\widetilde{\mathfrak{g}(0)}$, because there is an isomorphism of weight posets $\mathcal{P}\left(\mathbf{A}_{n-1}, 2 \varpi_{1}\right) \simeq \mathcal{P}\left(\mathbf{A}_{n}, \varpi_{2}\right)$, see [15, Theorem 2.1]. 
- For items 5 and 6 , the maximal rank level of $\Delta(1)$ is of size 2 . Hence deg $\mathcal{N}_{\Delta(1)}(t)=2$ and $\left[t^{2}\right] \mathcal{N}_{\Delta(1)}(t)$ is determined, because one knows $\mathcal{N}_{\Delta(1)}(1)=\# \mathfrak{A} \mathfrak{n}(\Delta(1))$.

- For item 7, there is a unique rank level of maximal size and its size is 3 . Therefore, $\operatorname{deg} \mathcal{N}_{\Delta(1)}(t)=3$ and $\left[t^{3}\right] \mathcal{N}_{\Delta(1)}(t)=1$, which again allows us to compute $\left[t^{2}\right] \mathcal{N}_{\Delta(1)}(t)$.

A posteriori, it is always true in the abelian case that

$$
\frac{\mathcal{N}_{\Delta(1)}^{\prime}(1)}{\mathcal{N}_{\Delta(1)}(1)}=\frac{\operatorname{dim} \mathfrak{g}(1)}{h}
$$

A relationship of this equality to conjectural properties of the reverse operator $\mathfrak{X}_{\Delta(1)}$ is discussed in Section 5.

Remark 3.5. The posets $\Delta(1)$ occurring in the abelian case are well known and usually called minuscule posets, see e.g. [21, 23, 24]. One can find several Hasse diagrams in [24, Appendix]. Since these posets are gaussian, there is a closed formula for the generating function for $m$-flags of order ideals in $\Delta(1)$ for any $m \in \mathbb{N}$ [21, Sect.6]; in particular, a formula for the $\mathcal{M}$-polynomial is obtained if $m=1$. Therefore, Theorem 3.3 is not really new. But our approach that exploits $\mathbb{Z}$-gradings, the Kostant-Macdonald identity, and the set of minimal length representatives $W^{0}$ seems to be new. It quickly provides a description of the upper (lower) ideals in $\Delta(1)$ and the corresponding antichains (Theorem 3.1). It can also be used for alternate proofs of other known results on $\mathfrak{A} \mathfrak{n}(\Delta(1))$. Anyway, our idea is that the minuscule posets should be treated as the simplest case of weight posets associated with $\mathbb{Z}$-gradings.

Recall that the abelian grading of $\mathfrak{g}$ related to an admissible $\alpha_{i} \in \Pi$ is defined by the minuscule weight $\varphi_{i}^{\vee}$ of the dual root system $\Delta^{\vee}$. Let $\mathcal{R}\left(\varphi_{i}^{\vee}\right)$ be the corresponding representation of the dual Lie algebra $\mathfrak{g}^{\vee}$ and $\mathcal{P}\left(\varphi_{i}^{\vee}\right)$ its weight poset.

Theorem 3.6 (cf. [21, Theorem 11]). The posets $(\mathfrak{A} \mathfrak{n}(\Delta(1)), \leqslant$ up $)$ and $\left(\mathcal{P}\left(\varphi_{i}^{\vee}\right), \preccurlyeq\right)$ are naturally isomorphic.

Proof. For a minuscule weight $\varphi_{i}^{\vee}$, it is known that $\mathcal{P}\left(\varphi_{i}^{\vee}\right)=W \varphi_{i}^{\vee}$ as a set [4, Ch. VIII, $\left.\S 7, \mathrm{n}^{0} 3\right]$. Since $W(0)$ is the stabiliser of $\varphi_{i}^{\vee}$, we have $\mathcal{P}\left(\varphi_{i}^{\vee}\right)=\left\{w\left(\varphi_{i}^{\vee}\right) \mid w \in W^{0}\right\}$. By Theorem 3.1, this yields the bijection $\Psi: \mathfrak{A} \mathfrak{n}(\Delta(1)) \rightarrow \mathcal{P}\left(\varphi_{i}^{\vee}\right), \Gamma_{w} \mapsto w\left(\varphi_{i}^{\vee}\right)$.

Let us prove that $\Psi$ respects the partial orders. Recall that the order ' $\leqslant$ up' corresponds to the inclusion of the corresponding upper ideals, see Section 2.

(a) Suppose that $\Gamma_{w}$ covers $\Gamma_{w^{\prime}}$ in $\mathfrak{A} \mathfrak{n}(\Delta(1))$ for some $w, w^{\prime} \in W^{0}$, i.e., $I_{w} \supset I_{w^{\prime}}$ and $\# I_{w}=\left(\# I_{w^{\prime}}\right)+1$. Then $\# N\left(w^{\prime}\right)=\# N(w)+1$ and $N\left(w^{\prime}\right)=N(w) \cup\{\gamma\}$, see Theorem 3.1(i). Therefore, $w^{\prime}=s_{\alpha} w$ for some $\alpha \in \Pi$ and hence $N\left(w^{\prime}\right)=N(w) \cup\left\{w^{-1}(\alpha)\right\}$. In particular, $\gamma=w^{-1}(\alpha) \in \Delta(1) \subset \Delta^{+}$. Next,

$$
w^{\prime}\left(\varphi_{i}^{\vee}\right)=s_{\alpha} w\left(\varphi_{i}^{\vee}\right)=w\left(\varphi_{i}^{\vee}\right)-\left(w\left(\varphi_{i}^{\vee}\right), \alpha\right) \alpha^{\vee}=w\left(\varphi_{i}^{\vee}\right)-\alpha^{\vee},
$$


since $\left(\varphi_{i}^{\vee}, w^{-1}(\alpha)\right)=1$. Whence $w\left(\varphi_{i}^{\vee}\right)-w^{\prime}\left(\varphi_{i}^{\vee}\right)=\alpha^{\vee} \in \Pi^{\vee}$, i.e., $w\left(\varphi_{i}^{\vee}\right)=\Psi\left(\Gamma_{w}\right)$ covers $w^{\prime}\left(\varphi_{i}^{\vee}\right)=\Psi\left(\Gamma_{w^{\prime}}\right)$ in $\mathcal{P}\left(\varphi_{i}^{\vee}\right)$

(b) Conversely, suppose that $w\left(\varphi_{i}^{\vee}\right)$ covers $w^{\prime}\left(\varphi_{i}^{\vee}\right)$ in $\mathcal{P}\left(\varphi_{i}^{\vee}\right)$ for some $w, w^{\prime} \in W^{0}$, i.e., $w\left(\varphi_{i}^{\vee}\right)-w^{\prime}\left(\varphi_{i}^{\vee}\right)=\alpha^{\vee} \in \Pi^{\vee}$. Then $\left(w\left(\varphi_{i}^{\vee}\right)-w^{\prime}\left(\varphi_{i}^{\vee}\right), \alpha\right)=2$, and the only possibility in the abelian setting is that $\left(w\left(\varphi_{i}^{\vee}\right), \alpha\right)=1$ and $\left(w^{\prime}\left(\varphi_{i}^{\vee}\right), \alpha\right)=-1$. It follows that $\left(\varphi_{i}^{\vee}, w^{-1}(\alpha)\right)=1$ and hence $w^{-1}(\alpha) \in \Delta(1) \subset \Delta^{+}$. Therefore, $\ell\left(s_{\alpha} w\right)=\ell(w)+1$ and $N\left(s_{\alpha} w\right)=N(w) \cup$ $\left\{w^{-1}(\alpha)\right\} \subset \Delta(1)$. Consequently, $s_{\alpha} w \in W^{0}$. Furthermore,

$$
s_{\alpha} w\left(\varphi_{i}^{\vee}\right)=w\left(\varphi_{i}^{\vee}\right)-\alpha^{\vee}=w^{\prime}\left(\varphi_{i}^{\vee}\right) .
$$

Whence $s_{\alpha} w=w^{\prime}$. This implies that $N\left(w^{\prime}\right)=N(w) \cup\left\{w^{-1}(\alpha)\right\}$. Thus, $I_{w}=I_{w^{\prime}} \cup\left\{w^{-1}(\alpha)\right\}$, and we are done.

Remark 3.7. Let ' $\leq$ ' denote the Bruhat order in $W$. It can be shown directly that the posets $\left(\mathfrak{A} \mathfrak{n}(\Delta(1)), \leqslant_{l o}\right)$ and $\left(W^{0}, \leq\right)$ are isomorphic. This yields an anti-isomorphism of $\left(W^{0}, \leq\right)$ and $\left(\mathcal{P}\left(\varphi_{i}^{\vee}\right), \preccurlyeq\right)$, which is a particular case of a general result of Proctor, see [20, Prop. 3]. (Note that Proctor's "Bruhat order" is opposite to the usual one!)

\section{ANTICHAINS AND UPPER IDEALS IN THE EXTRA-SPECIAL CASE}

In this section, we consider the extra-special $\mathbb{Z}$-grading of $\mathfrak{g}$. Now $\Delta^{+}=\Delta(0)^{+} \cup \Delta(1) \cup$ $\Delta(2), \Delta(2)=\{\theta\}$, and $\Delta(1)=\left\{\gamma \in \Delta \mid\left(\gamma, \theta^{\vee}\right)=1\right\}$ has the following obvious properties:

- If $\gamma_{1}, \gamma_{2} \in \Delta(1)$ and $\gamma_{1}+\gamma_{2}$ is a root, then $\gamma_{1}+\gamma_{2}=\theta$;

- The reflection $s_{\theta} \in W$ takes $\gamma \in \Delta(1)$ to $\gamma-\theta \in \Delta(-1)$. Hence $-s_{\theta}(\gamma) \in \Delta(1)$ and $\gamma+\left(-s_{\theta}(\gamma)\right)=\theta$

Let $h^{*}$ be the dual Coxeter number of $\Delta$. By definition, $h^{*}-1=\operatorname{ht}\left(\theta^{\vee}\right)$, the height of $\theta^{\vee}$ in $\Delta^{\vee}$. This implies that $h^{*} \leqslant h$, and $h=h^{*}$ if and only if all the roots have the same length. By [25], the total number of roots in $\Delta$ that are not orthogonal to $\theta$ is $4 h^{*}-6$. It follows that $\# \Delta(1)=\frac{1}{2}\left(4 h^{*}-6-2\right)=2 h^{*}-4$.

Definition 2. A subset $S \subset \Delta(1)$ is said to be Lagrangian, if $\# S=\frac{1}{2} \# \Delta(1)=h^{*}-2$ and there are no roots $\gamma_{1}, \gamma_{2} \in S$ such that $\gamma_{1}+\gamma_{2}=\theta$.

This terminology is justified by the fact that the $\mathfrak{t}$-stable subspace of $\mathfrak{g}(1)$ corresponding to such $S$ is Lagrangian w.r.t. the symplectic structure of $\mathfrak{g}(1)$ as $\mathfrak{g}(0)$-module.

As $\Delta(1)$ is the disjoint union of pairs $\{\gamma, \theta-\gamma\}=\left\{\gamma,-s_{\theta}(\gamma)\right\}, S$ is Lagrangian if and only if $\Delta(1) \backslash S$ is. Equivalently,

$S$ is Lagrangian if and only if $S=\Delta(1) \backslash-s_{\theta}(S)$.

Lemma 4.1. Let $I \subset \Delta(1)$ be an upper ideal.

(a) If $\gamma_{1}+\gamma_{2}=\theta$ for some $\gamma_{1}, \gamma_{2} \in I$, then there are no pairs $\mu_{1}, \mu_{2} \in \Delta(1) \backslash I$ such that $\mu_{1}+\mu_{2}=\theta$. In this case, $\# I>\frac{1}{2} \# \Delta(1)$. 
(b) If $\mu_{1}+\mu_{2}=\theta$ for some $\mu_{1}, \mu_{2} \in \Delta(1) \backslash I$, then there are no pairs $\gamma_{1}, \gamma_{2} \in I$ such that $\gamma_{1}+\gamma_{2}=\theta$. In this case, $\# I<\frac{1}{2} \# \Delta(1)$.

Proof. Assume that $\gamma_{1}+\gamma_{2}=\theta$ and $\mu_{1}+\mu_{2}=\theta$. Since $\left(\gamma_{1}+\gamma_{2}, \mu_{1}+\mu_{2}\right)>0$, we may conclude that, say, $\left(\gamma_{1}, \mu_{1}\right)>0$. Hence $\gamma_{1}-\mu_{1}=\mu_{2}-\gamma_{2} \in \Delta(0)$. Now, the assumption that $\mu_{2}-\gamma_{2} \in \Delta(0)^{+}$implies that $\mu_{2} \in I$; while the assumption that $\mu_{1}-\gamma_{1} \in \Delta(0)^{+}$implies that $\mu_{1} \in I$. These contradictions prove both (a) and (b).

In what follows, we have to keep track of the length of roots in $\Delta$. Write $\Delta_{l}\left(\right.$ resp. $\left.\Pi_{l}\right)$ for the set of all (resp. simple) long roots. In the ADE-case, all roots are assumed to be both long and short. Recall that the highest $\operatorname{root} \theta$ is always long and $\theta^{\vee}$ is always short.

Theorem 4.2. (i) There is a surjective map $\tau: W^{0} \rightarrow \mathfrak{A n}(\Delta(1)), w \mapsto \Gamma_{w}$, such that

$$
\# \tau^{-1}\left(\Gamma_{w}\right)= \begin{cases}1, & \text { if the upper ideal } I\left(\Gamma_{w}\right) \text { is not Lagrangian, } \\ 2, & \text { if the upper ideal } I\left(\Gamma_{w}\right) \text { is Lagrangian. }\end{cases}
$$

More precisely, $\Gamma_{w}=\min (\Delta(1) \backslash N(w))$ and $I_{w}:=I\left(\Gamma_{w}\right)=\Delta(1) \backslash N(w)$.

(ii) The upper ideal $I_{w}$ is Lagrangian if and only if $w(\theta) \in \pm \Pi_{l}$ if and only if $\tau^{-1}\left(\Gamma_{w}\right)=$ $\left\{w, w s_{\theta}\right\}$. In particular, $\# \mathfrak{A} \mathfrak{n}(\Delta(1))=\# W^{0}-\# \Pi_{l}=(h-1) \cdot \# \Pi_{l}$.

Proof. (i) If $w \in W^{0}$, then $N(w) \subset \Delta(1) \cup\{\theta\}$. Set $I_{w}:=\Delta(1) \backslash N(w)$ and $\Gamma_{w}:=\min \left(I_{w}\right)$. As in the proof of Theorem 3.1, one readily verifies that $I_{w}$ is an upper ideal of $\Delta(1)$. Hence $\Gamma_{w} \in \mathfrak{A} \mathfrak{n}(\Delta(1))$. This yields the mapping $\tau: W^{0} \rightarrow \mathfrak{A} \mathfrak{n}(\Delta(1))$ with $\tau(w)=\Gamma_{w}$. Let us prove that $\tau$ is onto and the fibres of $\tau$ match the above description.

Let $I \in \mathcal{J}_{+}(\Delta(1))$. To obtain $w \in W^{0}$ with $I=I_{w}$, we need two complementary closed subsets of $\Delta^{+}$such that one of them contains $\Delta(0)^{+} \cup I$, and the other contains $\Delta(1) \backslash I$. That is, the only problem is how to handle $\theta$. By Lemma 4.1, there are three possibilities:

(a) $\theta$ is a sum of two elements from $I$, but not from $\Delta(1) \backslash I$.

The only suitable pair of complementary closed subsets of $\Delta^{+}$is $\Delta(0)^{+} \cup I \cup\{\theta\}$ and $\Delta(1) \backslash I$. Here $N(w)=\Delta(1) \backslash I, \# I>\frac{1}{2} \# \Delta(1)$, and $w$ is the unique element of $W^{0}$ such that $I=I_{w}$.

(b) $\theta$ is a sum of two elements from $\Delta(1) \backslash I$, but not from $I$.

The only suitable pair of complementary closed subsets of $\Delta^{+}$is $\Delta(0)^{+} \cup I$ and $(\Delta(1) \backslash$ $I) \cup\{\theta\}$. Here $N(w)=(\Delta(1) \backslash I) \cup\{\theta\}, \# I<\frac{1}{2} \# \Delta(1)$, and $w$ is the unique element of $W^{0}$ such that $I=I_{w}$.

(c) $\theta$ cannot be written as a sum of two elements from either I or $\Delta(1) \backslash I$.

Then $I$ is Lagrangian and both $\Delta(1) \backslash I$ and $(\Delta(1) \backslash I) \cup\{\theta\}$ are the inversion sets of certain elements of $W^{0}$. That is, $\tau^{-1}(\min (I))$ consists of two elements.

This proves part (i).

(ii) Let $I \in \mathcal{J}_{+}(\Delta(1))$ be Lagrangian. Then $\tau^{-1}(\min (I))=\left\{w^{\prime}, w^{\prime \prime}\right\}$, where $N\left(w^{\prime}\right)=$ $\Delta(1) \backslash I$ and $N\left(w^{\prime \prime}\right)=(\Delta(1) \backslash I) \cup\{\theta\}$. We claim that $w^{\prime \prime}=w^{\prime} s_{\theta}$. Indeed, $s_{\theta}$ acts trivially 
on $\Delta(0)$ and therefore

$$
N\left(w^{\prime} s_{\theta}\right)=\left(\Delta(1) \backslash\left(-s_{\theta} N\left(w^{\prime}\right)\right) \cup\{\theta\}=N\left(w^{\prime}\right) \cup\{\theta\}=N\left(w^{\prime \prime}\right),\right.
$$

where the second equality holds because $N\left(w^{\prime}\right)$ is a Lagrangian subset, cf. Eq. (4.1). In particular, $w^{\prime}(\theta)=-w^{\prime \prime}(\theta)$, and our choice is that $w^{\prime}(\theta) \in \Delta^{+}$. If $w^{\prime}(\theta)=\delta_{1}+\delta_{2}$ is a sum of positive roots, then $\theta=w^{\prime-1}\left(\delta_{1}\right)+w^{\prime-1}\left(\delta_{2}\right)$, and the only possibility is that both roots $w^{\prime-1}\left(\delta_{1}\right), w^{\prime-1}\left(\delta_{2}\right)$ belong to $\Delta(1)$. It follows that $w^{\prime-1}\left(\delta_{1}\right), w^{\prime-1}\left(\delta_{2}\right) \in I=\Delta(1) \backslash N\left(w^{\prime}\right)$, which contradicts the fact that $I$ is Lagrangian. Thus, $w^{\prime}(\theta)$ must be a (long) simple root.

The above argument also shows that if $w \in W^{0}$ and $w(\theta) \notin \pm \Pi$, then $I_{w}$ cannot be Lagrangian. (More precisely, if $w(\theta) \in \Delta^{+} \backslash \Pi$, then one finds $\gamma_{1}, \gamma_{2} \in I_{w}$ such that $\gamma_{1}+\gamma_{2}=$ $\theta$; while if $w(\theta) \in-\left(\Delta^{+} \backslash \Pi\right)$, then one finds $\mu_{1}, \mu_{2} \in \Delta(1) \backslash I_{w}$ such that $\mu_{1}+\mu_{2}=\theta$.)

Since $W(0)$ is the stabiliser of $\theta$ in $W$, the mapping $W^{0} \rightarrow \Delta_{l}, w \mapsto w(\theta)$, is one-to-one. Consequently, there are exactly $\# \Pi_{l}$ Lagrangian ideals.

Finally, it follows from [3, Ch. VI, $\S 1.11$, Prop. 33] that the total number of long roots is $h \cdot \# \Pi_{l}$.

It is sometimes convenient to think of $\tau$ as the map from $W^{0}$ to $\mathcal{J}_{+}(\Delta(1))$ with $\tau(w)=$ $\Delta(1) \backslash N(w)$. As in the abelian case, we can give a characterisation of antichains associated with upper ideals in $\Delta(1)$ via the corresponding elements of $W^{0}$.

Theorem 4.3. For $I \in \mathcal{J}_{+}(\Delta(1))$, let $w_{I}$ be a corresponding element of $W^{0}$. (For the Lagrangian ideals $I$, we specify the choice of $w_{I}$ below.) Then

(i) $\gamma \in \min (I)$ if and only if $w_{I}(\gamma) \in \Pi$ (for a Lagrangian I, one has to choose $w_{I}$ such that $\left.w_{I}(\theta) \in-\Delta^{+}\right)$.

(ii) $\gamma \in \max (\Delta(1) \backslash I)$ if and only if $w_{I}(\gamma) \in-\Pi$ (for a Lagrangian $I$, one has to choose $w_{I}$ such that $\left.w_{I}(\theta) \in \Delta^{+}\right)$.

Proof. (i) If $\gamma \notin \min (I)$, then $\gamma=\gamma^{\prime}+\delta$ for some $\gamma^{\prime} \in I$ and $\delta \in \Delta(0)^{+}$. Whence $w_{I}(\gamma)=$ $w_{I}\left(\gamma^{\prime}\right)+w_{I}(\delta)$ is a sum of positive roots, i.e., $w_{I}(\gamma) \notin \Pi$ (for any choice of $w_{I}$ if $I$ is Lagrangian!).

Conversely, assume that $w_{I}(\gamma) \notin \Pi$, i.e., $w_{I}(\gamma)=\delta_{1}+\delta_{2}$ is a sum of positive roots. Then

$$
\gamma=w_{I}^{-1}\left(\delta_{1}\right)+w_{I}^{-1}\left(\delta_{2}\right) \in \Delta(1)
$$

Set $\mu_{i}=w_{I}^{-1}\left(\delta_{i}\right)$. There are two possibilities for $\mu_{1}, \mu_{2}$ :

(a) $\mu_{1} \in \Delta(0)$ and $\mu_{2} \in \Delta(1)$.

Then $\mu_{2} \in \Delta(1) \backslash N\left(w_{I}\right)=I$ and $\mu_{1} \in \Delta(0)^{+}$, since $w \in W^{0}$. Hence $\gamma=\mu_{1}+\mu_{2} \notin \min (I)$.

(b) $\mu_{1} \in \Delta(-1)$ and $\mu_{2}=\theta \in \Delta(2)$.

Then $w_{I}(\theta)=\delta_{2}$ is positive. Hence $\theta \notin N\left(w_{I}\right)$ and therefore $\# N\left(w_{I}\right) \leqslant \frac{1}{2} \# \Delta(1)$, i.e., $\# I \geqslant \frac{1}{2} \# \Delta(1)$.

- For $I$ Lagrangian, we agree to choose $w_{I}$ such that $w_{I}(\theta)<0$, which eliminates such a possibility for $\mu_{1}$ and $\mu_{2}$. 
- Assume that $\# I>\frac{1}{2} \# \Delta(1)$. We have $\gamma+\left(-\mu_{1}\right)=\theta$ and $-\mu_{1} \in \Delta(1) \backslash I$. If $\gamma \in \min (I)$, then $I^{\prime}:=I \backslash\{\gamma\}$ is again an upper ideal. Here $\gamma,-\mu_{1} \notin I^{\prime}$ and their sum is $\theta$. By Lemma 4.1(ii), we then have $(\# I)-1=\# I^{\prime}<\frac{1}{2} \# \Delta(1)$. And this contradicts the fact that $\# \Delta(1)$ is even.

Thus, $\gamma \notin \min (I)$ in all cases.

(ii) The proof here is similar and "dual" to the preceding part. For instance, at some point one refers to the "dual" fact that if $\gamma \in \max (\Delta(1) \backslash I)$, then $I \cup\{\gamma\}$ is again an upper ideal. The details are left to the reader.

Having computed the number of antichains (upper ideals) in $\Delta(1)$, we turn to computing the $t$-analogues $\mathcal{M}_{\Delta(1)}(t)$ and $\mathcal{N}_{\Delta(1)}(t)$. Although the relationship between the upper ideals of $\Delta(1)$ and $W^{0}$ appeared to be more involved in the extra-special case than in the abelian one, the formula for $\mathcal{M}_{\Delta(1)}(t)$ remains just the same!

Theorem 4.4. For the extra-special gradings, we have $\mathcal{M}_{\Delta(1)}(t)=\prod_{\gamma \in \Delta(1)} \frac{1-t^{\mathrm{ht}(\gamma)+1}}{1-t^{\mathrm{ht}(\gamma)}}$.

Proof. Let $P(t)$ denote the right hand side of the formula. A priori, $P(t)$ is only a rational function, and our first goal is to prove that $P(t)$ is a polynomial. Using the KostantMacdonald identity (cf. Theorem 3.3), the decomposition $\Delta^{+}=\Delta(0)^{+} \cup \Delta(1) \cup\{\theta\}$, and the equality $\mathrm{ht}(\theta)=h-1$, we obtain

$$
P(t)=\frac{\boldsymbol{W}(t)}{\boldsymbol{W}(\mathbf{0})(t)} \cdot \frac{1-t^{h-1}}{1-t^{h}}=\boldsymbol{W}^{\mathbf{0}}(t) \cdot \frac{1-t^{h-1}}{1-t^{h}}=\boldsymbol{W}^{\mathbf{0}}(t)-t^{h-1} \boldsymbol{W}^{\mathbf{0}}(t) \cdot \frac{1-t}{1-t^{h}} .
$$

It follows that $P(t) \in \mathbb{Z}[t]$ if and only if $\boldsymbol{W}^{\mathbf{0}}(t) \cdot \frac{1-t}{1-t^{h}} \in \mathbb{Z}[t]$. Recently, I proved that the latter is related to the Lusztig t-analogue of the zero weight multiplicity in $\mathcal{R}\left(\theta^{\vee}\right)$, the representation of the dual Lie algebra $\mathfrak{g}^{\vee}$ with highest weight $\theta^{\vee}$. Namely, let $\mathfrak{M}_{\theta^{\vee}}^{0}(t)$ be the above-mentioned $t$-analogue. It is a polynomial with nonnegative coefficients and $\mathfrak{M}_{\theta^{\vee}}^{0}(1)=m_{\theta^{\vee}}^{0}$, the respective weight multiplicity in $\mathcal{R}\left(\theta^{\vee}\right)$. By [17, Cor. 3.6], we have

$$
\boldsymbol{W}^{\mathbf{0}}(t) \cdot \frac{1-t}{1-t^{h}}=\frac{\mathfrak{M}_{\theta^{\vee}}^{0}(t)}{t^{h-h t\left(\theta^{\vee}\right)}}
$$

where $h t\left(\theta^{\vee}\right)$ is the height of $\theta^{\vee}$ in the dual root system $\Delta^{\vee}$. (Note that the indeterminate in [17] is denoted by $q$ in place of $t$ and our $\boldsymbol{W}^{0}(t)$ is $t_{0}(q) / t_{\theta}(q)$ therein.) As the left hand side has no pole at $t=0$, both parts are polynomials in $t$, which proves that $P(t)$ is a polynomial, too. Once we know that $P(t)$ is a polynomial, it follows from the very definition of it that $\operatorname{deg} P(t)=\# \Delta(1)=2 h^{*}-4$ and $P(t)$ is palindromic.

It remains to prove that $\left[t^{i}\right] P(t)$ is the number of upper ideals of cardinality $i$. Set $Q(t)=$ $\frac{\mathfrak{M}_{\theta^{\vee}}^{0}(t)}{t^{h-h t\left(\theta^{\vee}\right)}}$. (Although we do not need it directly, we note that $\operatorname{deg} \mathfrak{M}_{\theta^{\vee}}^{0}(t)=\mathrm{ht}\left(\theta^{\vee}\right)=h^{*}-1$, 
hence $\operatorname{deg} Q(t)=2 h^{*}-h-2$.) By Eq. (4.2) and (4.3), we have

$$
P(t)=\boldsymbol{W}^{\mathbf{0}}(t)-t^{h-1} Q(t) .
$$

As $h-1 \geqslant h^{*}-1$, it follows from Eq. (4.4) that

$$
\left[t^{i}\right] P(t)=\left[t^{i}\right] \boldsymbol{W}^{\mathbf{0}}(t)=\#\left\{w \in W^{0} \mid \ell(w)=i\right\} \quad \text { for } i \leqslant h^{*}-2 .
$$

If $w \in W^{0}$ and $\ell(w) \leqslant h^{*}-2=\frac{1}{2} \# \Delta(1)$, then Theorem 4.2 implies that $\theta \notin N(w)$ and $N(w) \subset \Delta(1)$. Hence for $i \leqslant h^{*}-2,\left[t^{i}\right] P(t)$ equals the number of upper ideals of cardinality $\# \Delta(1)-i$. Since $\mathcal{M}_{\Delta(1)}(t)$ is palindromic and of degree $\# \Delta(1)$ (Lemma 2.2), the latter is also the number of upper ideals of cardinality $i$. Thus, $\mathcal{M}_{\Delta(1)}(t)$ and $P(t)$ are palindromic of equal degrees $2 h^{*}-4$, and $\left[t^{i}\right] P(t)=\left[t^{i}\right] \mathcal{M}_{\Delta(1)}(t)$ for $i \leqslant h^{*}-2$. Consequently, $P(t) \equiv \mathcal{M}_{\Delta(1)}(t)$.

Corollary 4.5. In the extra-special case, we have $\# \mathfrak{A} \mathfrak{n}(\Delta(1))=\mathcal{M}_{\Delta(1)}(1)=\prod_{\gamma \in \Delta(1)} \frac{\mathrm{ht}(\gamma)+1}{\mathrm{ht}(\gamma)}$.

Remark 4.6. Yet another formula for $\mathcal{M}_{\Delta(1)}(t)$, which follows from Eq. (4.2) and (4.3), is

$$
\mathcal{M}_{\Delta(1)}(t)=\frac{\mathfrak{M}_{\theta \vee}^{0}(t)}{t^{h-\mathrm{ht}\left(\theta^{\vee}\right)}} \cdot \frac{1-t^{h-1}}{1-t} .
$$

The weight multiplicity $m_{\theta^{\vee}}^{0}=\mathfrak{M}_{\theta^{\vee}}^{0}(1)$ equals the number of short simple roots of $\mathfrak{g}^{\vee}$, i.e., the number of long simple roots of $\mathfrak{g}$, i.e., $\# \Pi_{l}$. Hence we again obtain the equality $\# \mathfrak{A} \mathfrak{n}(\Delta(1))=\mathcal{M}_{\Delta(1)}(1)=\# \Pi_{l} \cdot(h-1)$.

If $\Delta \in\{\mathbf{A D E}\}$, then $\mathfrak{g}^{\vee} \simeq \mathfrak{g}$, ht $\left(\theta^{\vee}\right)=h-1$, and $\mathfrak{M}_{\theta^{\vee}}^{0}(t)=\sum_{i=1}^{n} t^{m_{i}}$, where $m_{1}, \ldots, m_{n}$ are the exponents of $W$. Here we obtain a very simple explicit formula

$$
\mathcal{M}_{\Delta(1)}(t)=\left(\sum_{i=1}^{n} t^{m_{i}-1}\right)\left(1+t+\ldots+t^{h-2}\right) .
$$

Theorem 4.7. In the extra-special case, $\operatorname{deg} \mathcal{N}_{\Delta(1)}(t) \leqslant 3$, i.e., if $\Gamma \in \mathfrak{A} \mathfrak{n}(\Delta(1))$, then $\# \Gamma \leqslant 3$.

Proof. Recall that $\mathfrak{g}(1)$ is a simple $\mathfrak{g}(0)$-module unless $\Delta$ is of type $\mathbf{A}_{n}$, and therefore in all these cases $\Delta(1)$ is rank symmetric, rank unimodal, and Sperner (see Lemma 2.6). Therefore, $\operatorname{deg} \mathcal{N}_{\Delta(1)}(t)=\# \Delta(1)_{i}$, where $\Delta(1)_{i}$ is the set of roots of height $i$ in $\Delta(1)$ and $i$ is a middle rank of $\Delta(1)$. As $h t(\theta)=h-1$ and the unique element of $\Delta^{+}$covered by $\theta$ belongs to $\Delta(1)$, the roots in $\Delta(1)$ have the height between 1 and $h-2$. Furthermore, $h$ is even, if $\mathfrak{g}$ is not of type $\mathbf{A}_{n}$. Hence the two middle ranks are $(h-2) / 2$ and $h / 2$, and $\operatorname{deg} \mathcal{N}_{\Delta(1)}(t)=\# \Delta(1)_{h / 2}=\# \Delta(1)_{(h-2) / 2}$. Assume that $\Delta(1)_{h / 2}=\left\{\gamma_{1}, \ldots, \gamma_{k}\right\}$. Since $\gamma_{i} \pm \gamma_{j}$ is not a root, all roots in $\Delta(1)_{h / 2}$ are pairwise orthogonal. Consequently, $\mu:=\theta-\sum_{i=1}^{k} \gamma_{i}$ is a root. Because $\theta \in \Delta(2)$ and $\gamma_{i} \in \Delta(1)$, we have $\mu \in \Delta(2-k)$. Therefore, $k \leqslant 4$, and if $k=4$, then $\mu=-\theta$. However, comparing heights in the equality $2 \theta=\sum_{i=1}^{4} \gamma_{i}$, we see that this is impossible! (For, $2 \mathrm{ht}(\theta)=2 h-2$, whereas $\sum_{i=1}^{4} \mathrm{ht}\left(\gamma_{i}\right)=2 h$.) Thus, $k \leqslant 3$.

For $\mathbf{A}_{n}$, the poset $\Delta(1)$ is the disjoint union of two chains, $\Delta(1)=\mathfrak{C}_{n-1} \sqcup \mathfrak{C}_{n-1}$, whence $\operatorname{deg} \mathcal{N}_{\Delta(1)}(t)=2$. 
Thus, $\mathcal{N}_{\Delta(1)}(t)=1+N_{1} t+N_{2} t^{2}+N_{3} t^{3}, N_{1}=\# \Delta(1)$, and $\mathcal{N}_{\Delta(1)}(1)=\# \mathfrak{A} \mathfrak{n}(\Delta(1))$. Therefore, to completely determine $\mathcal{N}_{\Delta(1)}(t)$, only one more condition is needed. Below, we compute $N_{2}$ in the ADE-case and thereby provide a nice uniform expression for $\mathcal{N}_{\Delta(1)}(t)$. To this end, we begin with a general look at the two-element antichains in $\Delta(1)$ (=2-antichains).

Let $\left\{\gamma_{1}, \gamma_{2}\right\}$ be a 2-antichain in $\Delta(1)$. Then $\left(\gamma_{1}, \gamma_{2}\right) \leqslant 0$ and there are two possibilities:

$\left(a_{1}\right)\left(\gamma_{1}, \gamma_{2}\right)=0$. Such an antichain is said to be orthogonal.

$\left(a_{2}\right)\left(\gamma_{1}, \gamma_{2}\right)<0$. Then $\gamma_{1}+\gamma_{2}=\theta$ and such an antichain is said to be summable.

If $\left\{\gamma_{1}, \gamma_{2}\right\}$ is an orthogonal antichain, then $\gamma_{1}+\gamma_{2}-\theta$ is a root, necessarily in $\Delta(0)$; while in the second case, $\gamma_{1}+\gamma_{2}-\theta=0$. Therefore, one obtains a general map

$$
\varkappa: \mathfrak{A} \mathfrak{n}(\Delta(1))_{\langle 2\rangle} \rightarrow \Delta(0) \cup\{0\}
$$

where $\mathfrak{A} \mathfrak{n}(\Delta(1))_{\langle 2\rangle}$ is the set of 2-antichains in $\Delta(1)$ and $\varkappa\left(\left\{\gamma_{1}, \gamma_{2}\right\}\right)=\gamma_{1}+\gamma_{2}-\theta$.

Theorem 4.8. If $\Delta \in\{\mathrm{ADE}\}$, then (i) for any $\mu \in \Delta(0)$, there is a unique (orthogonal) 2antichain $\Gamma$ such that $\varkappa(\Gamma)=\mu$, (ii) the number of summable antichains is equal to $\mathrm{rk} \mathfrak{g}-1$. In particular, $\varkappa$ is onto and $N_{2}=\operatorname{dim} \mathfrak{g}(0)-1=\operatorname{dim} \widetilde{\mathfrak{g}(0)}$.

Proof. The argument below is not entirely case-free. On the other hand, a complete caseby-case checking is also possible. For this reason, we only outline some steps and their status (case-free or case-by-case).

1. If $\left\{\gamma_{1}, \gamma_{2}\right\}$ is an antichain in $\Delta(1)$, then so is $\left\{\theta-\gamma_{1}, \theta-\gamma_{2}\right\}$, and $\varkappa\left(\left\{\gamma_{1}, \gamma_{2}\right\}\right)=$ $-\varkappa\left(\left\{\theta-\gamma_{1}, \theta-\gamma_{2}\right\}\right)$. Therefore $\mu \in \operatorname{Im}(\varkappa)$ if and only if $-\mu \in \operatorname{Im}(\varkappa)$, and it suffices to consider only $\mu \in \Delta(0)^{+} \cup\{0\}$.

2. (Uniqueness) Assume that $\left\{\gamma_{1}, \gamma_{2}\right\}$ and $\left\{\gamma_{1}^{\prime}, \gamma_{2}^{\prime}\right\}$ lie in $\varkappa^{-1}(\mu)$, i.e., $\gamma_{1}+\gamma_{2}=\gamma_{1}^{\prime}+\gamma_{2}^{\prime}=$ $\theta+\mu$ for some $\mu \in \Delta(0)^{+}$. All the roots involved have the same length and $\left(\gamma_{1}, \gamma_{2}\right)=$ $\left(\gamma_{1}^{\prime}, \gamma_{2}^{\prime}\right)=0$. Since $\left(\gamma_{1}, \gamma_{1}\right)+\left(\gamma_{2}, \gamma_{2}\right)=\left(\gamma_{1}+\gamma_{2}\right)^{2}=\left(\gamma_{1}+\gamma_{2}, \gamma_{1}^{\prime}+\gamma_{2}^{\prime}\right)$, we have $\left(\gamma_{i}, \gamma_{j}^{\prime}\right)>0$ for all $i, j \in\{1,2\}$. In particular, $\gamma_{i}-\gamma_{j}^{\prime} \in \Delta(0)$. Set $\nu=\gamma_{1}^{\prime}-\gamma_{1}$ and $\eta=\gamma_{1}^{\prime}-\gamma_{2}=\gamma_{1}-\gamma_{2}^{\prime}$. W.l.o.g. we may assume that $\nu \in \Delta(0)^{+}$, hence $\gamma_{1}^{\prime} \succcurlyeq \gamma_{1}$ in $\Delta(1)$. Now, if $\eta \in \Delta(0)^{+}$, then $\gamma_{1}^{\prime} \succcurlyeq \gamma_{1} \succcurlyeq \gamma_{2}^{\prime}$, i.e., $\left\{\gamma_{1}^{\prime}, \gamma_{2}^{\prime}\right\}$ is not an antichain; if $-\eta \in \Delta(0)^{+}$, then $\gamma_{2} \succcurlyeq \gamma_{1}^{\prime} \succcurlyeq \gamma_{1}$, i.e., $\left\{\gamma_{1}, \gamma_{2}\right\}$ is not an antichain. These contradictions prove that $\# \varkappa^{-1}(\mu) \leqslant 1$.

3. (Existence) (i) (Base) if $\mu$ is the highest root of an irreducible subsystem of $\Delta(0)$, then $\varkappa^{-1}(\mu) \neq \varnothing$. [case-by-case]

(ii) (Induction step) if $\varkappa^{-1}(\mu) \neq \varnothing$ and $\mu-\alpha \in \Delta(0)^{+} \cup\{0\}$ for some $\alpha \in \Pi(0)$, then $\varkappa^{-1}(\mu-\alpha) \neq \varnothing$. [case-free]

These three steps prove everything concerning the orthogonal antichains, and also show that summable antichains exist; hence $\varkappa$ is onto.

4. The assertion on the number of summable antichains occurs as a by-product of certain results of mine related to abelian $\mathfrak{b}$-ideals in $\mathfrak{u}^{+}$. This will appear elsewhere. Actually, 
those results provide a one-to-one correspondence between the summable antichains and the edges of the Dynkin diagram. [case-free]

This completes our outline.

As an illustration to the proof, we point out all summable antichains for $\mathbf{E}_{6}$. The numbering of simple roots is $\begin{gathered}1-2-3-4-5 \\ \frac{1}{6}\end{gathered}$ and $\left(n_{1} n_{2} \ldots n_{6}\right)$ stands for the root $\gamma=\sum_{i=1}^{6} n_{i} \alpha_{i}$. In particular, $\theta=(123212)$ and $\gamma \in \Delta(1)$ if and only if $n_{6}=1$. Then the summable antichains in $\Delta(1)$ are:

$\{111001,012211\},\{111101,012111\},\{111111,012101\},\{011111,112101\},\{001111,122101\}$.

Note also that for $\mathbf{A}_{n}$ and $\mathbf{D}_{n}$, everything in Theorem 4.8 can explicitly be verified, using the usual $\left\{\varepsilon_{i}\right\}$ presentation of the roots.

Corollary 4.9. If $\Delta \in\{\mathrm{ADE}\}$, then

$$
\mathcal{N}_{\Delta(1)}(t)=1+\operatorname{dim} \mathfrak{g}(1) \cdot t+(\operatorname{dim} \mathfrak{g}(0)-1) \cdot t^{2}+(\operatorname{dim} \mathfrak{g}(1)-2 \mathrm{rk} \mathfrak{g}+2) \cdot t^{3} .
$$

For $\mathbf{A}_{n}$, we have $\operatorname{dim} \mathfrak{g}(1)=2 \mathrm{rkg}-2=2 n-2$ and $\operatorname{deg} \mathcal{N}_{\Delta(1)}(t)=2$ (if $n>1$ ). It also follows from Eq. (4.5) that $\frac{\mathcal{N}_{\Delta(1)}^{\prime}(1)}{\mathcal{N}_{\Delta(1)}(1)}=\frac{2 \operatorname{dim} \mathfrak{g}-6 \mathrm{rk} \mathfrak{g}}{\operatorname{dim} \mathfrak{g}-2 \mathrm{rkg}}=\frac{2 h-4}{h-1}$, since $\operatorname{dim} \mathfrak{g}=(h+1) \mathrm{rk} \mathfrak{g}$.

Remark. Some steps in the proof of Theorem 4.8 go through for any $\Delta$ (e.g. 1. and 2.), but $\varkappa$ is no longer onto in general. Although $\operatorname{Im}(\varkappa)$ can explicitly be described in each non-simply laced case, we are unable to infer from it a general characterisation of $\operatorname{Im}(\varkappa)$. Here is the list of $\mathcal{N}$-polynomials for the remaining root systems:

$$
\begin{aligned}
& \mathbf{B}_{n}, n \geqslant 2: \quad \mathcal{N}_{\Delta(1)}(t)=1+2(2 n-3) t+(n-2)(2 n-3) t^{2} ; \\
& \mathbf{C}_{n}, n \geqslant 2: \quad \mathcal{N}_{\Delta(1)}(t)=1+(2 n-2) t ; \\
& \mathbf{F}_{4}: \quad \mathcal{N}_{\Delta(1)}(t)=1+14 t+7 t^{2} ; \\
& \mathbf{G}_{2}: \quad \mathcal{N}_{\Delta(1)}(t)=1+4 t ;
\end{aligned}
$$

A posteriori, for all irreducible root systems $\Delta$, we have

$$
\frac{\mathcal{N}_{\Delta(1)}^{\prime}(1)}{\mathcal{N}_{\Delta(1)}(1)}=\frac{\# \Delta(1)}{h-1}=\frac{2 h^{*}-4}{h-1}
$$

As in the abelian case (cf. Theorem 3.6), we provide a realisation of $\left(\mathfrak{A n}(\Delta(1)), \leqslant_{u p}\right)$ via the representation of $\mathfrak{g}^{\vee}$ associated with the defining element of the $\mathbb{Z}$-grading, which is now $\theta^{\vee} \in \Delta^{\vee}$. A new phenomenon is that $\mathcal{R}\left(\theta^{\vee}\right)$ is not wmf. Indeed, $\mathcal{P}\left(\theta^{\vee}\right)=\{0\} \cup\left(\Delta_{l}\right)^{\vee}$, i.e., it contains zero and the short roots of $\Delta^{\vee}$. The multiplicity of 0 equals $\# \Pi_{l}$, i.e., the number of short simple roots in $\Delta^{\vee}$, and all other weights are of multiplicity one (and form a sole $W$-orbit). The number of nonzero weights is $\# W\left(\theta^{\vee}\right)=\# W^{0}=h \cdot \# \Pi_{l}$. To adjust it to the relationship between $\mathfrak{A} \mathfrak{n}(\Delta(1))$ and $W^{0}$ occurring in Theorem 4.2, we do the following:

- forget about $0 \in \mathcal{P}\left(\theta^{\vee}\right)$, i.e., stick to $\left(\Delta_{l}\right)^{\vee}$; 
- identify $\alpha^{\vee}$ and $-\alpha^{\vee}$ in $\left(\Delta_{l}\right)^{\vee}$ for $\alpha \in \Pi_{l}$.

The resulting set is denoted by $\left(\Delta_{l}\right)^{\vee} / \sim$. It has the natural partial order induced from $\left(\mathcal{P}\left(\theta^{\vee}\right), \preccurlyeq\right)$. For, we change nothing in the upper (positive) part $\left(\Delta_{l}^{+}\right)^{\vee}$ and in the lower (negative) part $-\left(\Delta_{l}^{+}\right)^{\vee}$ of $\mathcal{P}\left(\theta^{\vee}\right)$. And we only identify "element-wise" the subsets $\min \left(\left(\Delta_{l}^{+}\right)^{\vee}\right)=\left(\Pi_{l}\right)^{\vee}$ and $\max \left(-\left(\Delta_{l}^{+}\right)^{\vee}\right)=\left(-\Pi_{l}\right)^{\vee}$.

Theorem 4.10. The posets $(\mathfrak{A} \mathfrak{n}(\Delta(1)), \leqslant u p)$ and $\left(\left(\Delta_{l}\right)^{\vee} / \sim, \preccurlyeq\right)$ are naturally isomorphic.

Proof. In Theorem 4.2, we have defined the surjective map $\tau: W^{0} \rightarrow \mathfrak{A} \mathfrak{n}(\Delta(1))$. Recall that $\# \tau^{-1}(\Gamma) \leqslant 2$ and $\# \tau^{-1}(\Gamma)=2$ if and only if $I(\Gamma) \in \mathcal{J}_{+}(\Delta(1))$ is Lagrangian. Moreover, if $\tau^{-1}(\Gamma)=\left\{w^{\prime}, w^{\prime \prime}\right\}$, then $w^{\prime}(\theta)=-w^{\prime \prime}(\theta) \in \pm \Pi_{l}$. Therefore,

- if $I(\Gamma)$ is Lagrangian, then $\tau^{-1}(\Gamma)(\theta)=\{\alpha,-\alpha\}$ for some $\alpha \in \Pi_{l}$;

- if $I(\Gamma)$ is not Lagrangian, then $\tau^{-1}(\Gamma)(\theta)=\mu \in \Delta_{l} \backslash(\Pi \cup-\Pi)$.

Thus, the map $\Psi: \mathfrak{A} \mathfrak{n}(\Delta(1)) \rightarrow\left(\Delta_{l}\right)^{\vee} / \sim, \Gamma \mapsto \tau^{-1}(\Gamma)(\theta)$, is well-defined and onto. Let us prove that $\Psi$ respects the partial orders.

(a) Suppose that $\Gamma_{1}$ covers $\Gamma_{2}$ in $\left(\mathfrak{A n}(\Delta(1))\right.$, $\left.\leqslant_{u p}\right)$, i.e., $I\left(\Gamma_{1}\right)=I\left(\Gamma_{2}\right) \cup\{\gamma\}$ for some $\gamma \in \Delta(1)$. Our goal is to obtain the relation $N\left(w_{2}\right)=N\left(w_{1}\right) \cup\{\gamma\}$ for some $w_{i} \in \tau^{-1}\left(\Gamma_{i}\right)$. If this is the case, then $w_{2}=s_{\alpha} w_{1}$ and $\gamma=w_{1}^{-1}(\alpha)$ for some $\alpha \in \Pi$. Furthermore,

$$
w_{2}\left(\theta^{\vee}\right)=s_{\alpha} w_{1}\left(\theta^{\vee}\right)=w_{1}\left(\theta^{\vee}\right)-\left(w_{1}\left(\theta^{\vee}\right), \alpha\right) \alpha^{\vee}=w_{1}\left(\theta^{\vee}\right)-\alpha^{\vee},
$$

which implies that $\Psi\left(\Gamma_{1}\right)$ covers $\Psi\left(\Gamma_{2}\right)$ in $\left(\Delta_{l}\right)^{\vee} / \sim$. It is important here that both $w_{1}\left(\theta^{\vee}\right)$ and $w_{2}\left(\theta^{\vee}\right)$ lie in either $\left(\Delta_{l}^{+}\right)^{\vee}$ or $\left(-\Delta_{l}^{+}\right)^{\vee}$.

\section{How to reach that goal:}

$\left(\mathbf{a}_{1}\right)$ If neither $I\left(\Gamma_{1}\right)$ nor $I\left(\Gamma_{2}\right)$ is Lagrangian, then $w_{1}, w_{2}$ are uniquely determined and the required relation holds automatically. In particular, if $\# I\left(\Gamma_{1}\right)<\frac{1}{2} \# \Delta(1)$, then both $N\left(w_{1}\right)$ and $N\left(w_{2}\right)$ contain $\theta$ and $w_{1}\left(\theta^{\vee}\right), w_{2}\left(\theta^{\vee}\right) \in\left(\Delta_{l}^{+}\right)^{\vee}$; while if $\# I\left(\Gamma_{2}\right)>\frac{1}{2} \# \Delta(1)$, then both $N\left(w_{1}\right)$ and $N\left(w_{2}\right)$ do not contain $\theta$ and $w_{1}\left(\theta^{\vee}\right), w_{2}\left(\theta^{\vee}\right) \in\left(-\Delta_{l}^{+}\right)^{\vee}$.

$\left(\mathbf{a}_{2}\right)$ If one of the ideals is Lagrangian (note that there are two different possibilities for this), then the "non-Lagrangian" element $w_{i}$ is uniquely determined, and for the Lagrangian ideal $I\left(\Gamma_{\bar{i}}\right)$ we choose the element $w_{\bar{i}} \in \tau^{-1}\left(\Gamma_{\bar{i}}\right)$ such that $w_{i}(\theta)$ and $w_{\bar{i}}(\theta)$ have the same sign. This choice guarantee us that $N\left(w_{i}\right)$ and $N\left(w_{\bar{i}}\right)$ simultaneously contain or do not contain $\theta$.

(b) Conversely, if $w\left(\varphi_{i}^{\vee}\right)$ covers $w^{\prime}\left(\varphi_{i}^{\vee}\right)$ in $\left(\Delta_{l}\right)^{\vee} / \sim$, then, as in part (a), the argument goes through along the lines presented in Theorem 3.6, with amendments caused by the presence of $\{\theta\}=\Delta(2)$ and relation ' $\sim$ ' in $\left(\Delta_{l}\right)^{\vee}$. We omit the details.

\section{CONJECTURES AND EXAMPLES}

So far, almost nothing is said about the reverse operators for posets $\Delta(1)$. This will be fixed below. Numerous calculations performed in the abelian, extra-special, and some other cases suggest that the reverse operators $\mathfrak{X}_{\Delta(1)}$ have very good properties similar to 
those of $\mathfrak{X}_{\Delta^{+}}$(see Introduction), and also a new one. But outside the realm of the wmf representations associated with $\mathbb{Z}$-gradings some of these properties certainly fail.

Conjecture 5.1. For any $\mathbb{Z}$-grading of $\mathfrak{g}$, we have $\mathcal{M}_{\Delta(1)}(t)=\prod_{\gamma \in \Delta(1)} \frac{1-t^{\mathrm{ht}(\gamma)+1}}{1-t^{\mathrm{ht}(\gamma)}}$. In particular, $\# \mathfrak{A} \mathfrak{n}(\Delta(1))=\mathcal{M}_{\Delta(1)}(1)=\prod_{\gamma \in \Delta(1)} \frac{\mathrm{ht}(\gamma)+1}{\mathrm{ht}(\gamma)}$.

This conjecture readily reduces to 1 -standard gradings. First, the $\mathfrak{g}(0)$-module $\mathfrak{g}(1)$ is determined by $\Pi(1)$ and does not depend on $\Pi(\geqslant 2)$. Having removed from the Dynkin diagram the nodes (simple roots) in $\Pi(\geqslant 2)$, we get a standard $\mathbb{Z}$-grading of a semisimple subalgebra $\mathfrak{s} \subset \mathfrak{g}$, with the same poset $\Delta(1)$. Second, each simple $\mathfrak{s}(0)$-submodule of $\mathfrak{s}(1)$ is associated with a 1 -standard $\mathbb{Z}$-grading of a certain simple factor of $\mathfrak{s}$, and we can use the relevant assertion of Lemma 2.1.

In view of Theorems 3.3 and 4.4, Conjecture 5.1 holds for the abelian and extra-special gradings. Furthermore, it is true if $\Delta(1)$ is the direct product of at most three chains, see Remark 5.8. This covers all but one 1-standard $\mathbb{Z}$-gradings for series $\mathbf{A}_{n}, \mathbf{B}_{n}$, and $\mathbf{C}_{n}$, see Example 5.6. Conjecture 5.1 has also a natural counterpart for arbitrary irreducible wmf representations. If $\mathcal{P}(V)$ is the weight poset of an irreducible wmf representation $V$ and $r: \mathcal{P}(V) \rightarrow \mathbb{N}$ is the tuned rank function, then one might suggest that

$$
\mathcal{M}_{\mathcal{P}(V)}(t)=\prod_{\nu \in \mathcal{P}(V)} \frac{1-t^{r(\nu)+1}}{1-t^{r(\nu)}} \text { and hence } \# \mathcal{P}(V)=\prod_{\nu \in \mathcal{P}(V)} \frac{r(\nu)+1}{r(\nu)} .
$$

However, it can happen for some $V$ that the first product is not a polynomial and the second product in not an integer, see Example 5.13(1) below.

An interesting feature of the polynomials $\mathcal{M}_{\Delta(1)}(t)$ is that they seem to provide a nice illustration to the " $t=-1$ phenomenon" of Stembridge [24], which is a particular case of the cyclic sieving phenomenon [22]. Recall that, for any $I \in \mathcal{J}_{+}(\Delta(1))$, we have defined the dual ideal $I^{*}=\Delta(1) \backslash w_{0}(I)$, where $w_{0} \in W(0)$ is the longest element.

Conjecture 5.2. The value $\mathcal{M}_{\Delta(1)}(-1)$ equals the number of upper ideals I such that $I^{*}=I$.

Results of [24] confirm this assertion in the abelian case. Using the formula in Remark 4.6, we can also prove it in the extra-special case. But, the main challenge is to provide conceptual proofs for all (at least, some of) the previous and subsequent conjectures!

Conjecture 5.3. Let $\mathfrak{g}=\bigoplus_{i \in \mathbb{Z}} \mathfrak{g}(i)$ be a 1-standard $\mathbb{Z}$-grading (hence $\mathfrak{g}(1)$ is a simple $\mathfrak{g}(0)$ module and $\mathfrak{g}(0)$ is semisimple).

(i) If $d_{1}=\max \{\mathrm{ht}(\gamma) \mid \gamma \in \Delta(1)\}$, then $\operatorname{ord}\left(\mathfrak{X}_{\Delta(1)}\right)=d_{1}+1$.

(ii) the average value of the size of antichains in any $\mathfrak{X}_{\Delta(1)}$-orbit is the same and equals $\frac{\operatorname{dim} \mathfrak{g}(1)}{d_{1}+1}=\frac{\# \Delta(1)}{\operatorname{ord}\left(\mathfrak{X}_{\Delta(1)}\right)} ;$ 
(iii) the average value of the size of upper ideals in any $\mathfrak{X}_{\Delta(1)}$-orbit is the same and equals $\operatorname{dim} \mathfrak{g}(1) / 2$.

Part (iii) above is a new property that has no counterpart for $\mathcal{P}=\Delta^{+}$.

In the abelian (resp. extra-special) case, we have $d_{1}=h-1$ (resp. $d_{1}=h-2$ ). Therefore, Conjecture 5.3(i) claims that in these cases ord $\left(\mathfrak{X}_{\Delta(1)}\right)$ is equal to $h$ and $h-1$, respectively.

Example 5.4. (cf. [14, Lemma 1.1]) Let $\mathcal{P}=\bigsqcup_{j=1}^{d} \mathcal{P}_{i}$ be a graded poset such that $\max (\mathcal{P})=$ $\mathcal{P}_{d}$ and $\min (\mathcal{P})=\mathcal{P}_{1}$, then $\mathfrak{X}_{\mathcal{P}}$ always has an orbit of size $d+1$. Namely, we have $\mathfrak{X}_{\mathcal{P}}\left(\mathcal{P}_{i}\right)=$ $\mathcal{P}_{i-1}$ (with $\mathcal{P}_{0}=\varnothing$ ) and $\left\{\varnothing, \mathcal{P}_{d}, \ldots, \mathcal{P}_{1}\right\}$ is an $\mathfrak{X}_{\mathcal{P}}$-orbit. Moreover, the average value of the size of antichains in this orbit equals $\# \mathcal{P} /(d+1)$. Even more, if $\mathcal{P}$ is rank symmetric, then the average value of the size of upper ideals in this orbit equals $\# \mathcal{P} / 2$.

Since all these properties hold for $\mathcal{P}=\Delta(1)$, we get a motivating example for the whole Conjecture 5.3.

Example 5.5. Straightforward computations for some abelian gradings show that

- For $\mathfrak{g}=\mathbf{E}_{6}$ and $\Pi(1)=\left\{\alpha_{1}\right\}$ (item 6 in Section 3), $\mathfrak{X}_{\Delta(1)}$ has three orbits of sizes $12,12,3$. We also know here that $\# \mathfrak{A} \mathfrak{n}(\Delta(1))=27$. Hence $\operatorname{ord}\left(\mathfrak{X}_{\Delta(1)}\right)=12$.

- For $\mathfrak{g}=\mathbf{E}_{7}$ and $\Pi(1)=\left\{\alpha_{1}\right\}$ (item 7 in Section 3 ), $\mathfrak{X}_{\Delta(1)}$ has four orbits of sizes $18,18,18,2$. We also know here that $\# \mathfrak{A} \mathfrak{n}(\Delta(1))=56$. Hence ord $\left(\mathfrak{X}_{\Delta(1)}\right)=18$.

- For $\mathfrak{g}=\mathbf{D}_{n}$ and $\Pi(1)=\left\{\alpha_{1}\right\}$ (item 5 in Section 3), we denote $\Delta(1)$ by $\mathcal{D}_{n-1}$. The corresponding Hasse diagram is

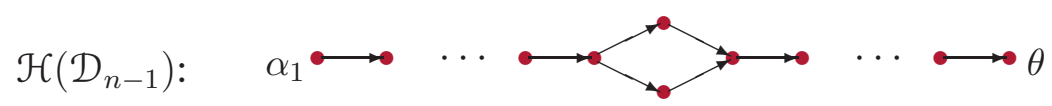

(The number of nodes is $2 n-2$.) Here $\# \mathfrak{A} \mathfrak{n}(\Delta(1))=2 n$ and $\mathfrak{X}_{\Delta(1)}$ has two orbits of sizes 2 and $2 n-2$, i.e., $\operatorname{ord}\left(\mathfrak{X}_{\Delta(1)}\right)=2 n-2$.

The other assertions of Conjecture 5.3 are also satisfied in these three cases.

Example 5.6. Recall that $\mathcal{C}_{k}$ is a $k$-element chain. For $\mathcal{P}=\mathcal{C}_{k} \times \mathcal{C}_{m}$, Fon-der-Flaass proved that $\operatorname{ord}\left(\mathfrak{X}_{\mathcal{P}}\right)=k+m$, see [9, Theorem 2]. This confirms Conjecture 5.3(i) for all 1-standard $\mathbb{Z}$-gradings with $\Delta(1) \simeq \mathfrak{C}_{k} \times \mathfrak{C}_{m}$, because it is then clear that the highest weight of $\Delta(1)$ (= the root of maximal height) has height $k+m-1$. In particular, this happens for all but one 1-standard $\mathbb{Z}$-gradings in types $\mathbf{A}_{n}, \mathbf{B}_{n}$, and $\mathbf{C}_{n}$. More precisely,

- If $\Pi(1)=\left\{\alpha_{i}\right\}$ for $\mathbf{A}_{n}$, then $\Delta(1) \simeq \mathcal{C}_{i} \times \mathcal{C}_{n+1-i}$ and the highest weight in $\Delta(1)$ is $\theta$.

- If $\Pi(1)=\left\{\alpha_{i}\right\}$ for $\mathbf{B}_{n}$, then $\Delta(1) \simeq \mathfrak{C}_{i} \times \mathfrak{C}_{2 n+1-2 i}$.

- If $\Pi(1)=\left\{\alpha_{i}\right\}$ for $\mathbf{C}_{n}$ and $i<n$, then $\Delta(1) \simeq \mathcal{C}_{i} \times \mathcal{C}_{2 n-2 i}$.

However, $\Delta(1)$ has another structure for the remaining case in $\mathbf{C}_{n}$ and all 1-standard gradings of $\mathbf{D}_{n}$. If $\Pi(1)=\left\{\alpha_{i}\right\}$ for $\mathbf{D}_{n}$ and $i \leqslant n-2$, then $\Delta(1) \simeq \mathcal{C}_{i} \times \mathcal{D}_{n-i}$. Note, however, that $\mathcal{D}_{2} \simeq \mathcal{C}_{2} \times \mathcal{C}_{2}$. 
Example 5.7. For the poset $\mathcal{C}_{k} \times \mathcal{C}_{m} \times \mathcal{C}_{n}=: \mathcal{C}_{(k, m, n)}$, it is proved in [5, Theorem 6(b)] that $\operatorname{ord}(\mathfrak{X})=k+m+n-1$ if $k=2$. (The general case is open!) In real life, posets of the form $\mathcal{C}_{(2, m, n)}$ occur in connection with 1-standard $\mathbb{Z}$-gradings corresponding to the branching node of the Dynkin diagram for $\mathbf{D}_{n}$ or $\mathbf{E}_{n}$. Namely,

- If $\Pi(1)=\left\{\alpha_{n-2}\right\}$ for $\mathbf{D}_{n}$, then $\Delta(1) \simeq \mathfrak{C}_{(2,2, n-2)}$;

- If $\Pi(1)=\left\{\alpha_{3}\right\}$ for $\mathbf{E}_{6}$, then $\Delta(1) \simeq \mathfrak{C}_{(2,3,3)}$;

- If $\Pi(1)=\left\{\alpha_{4}\right\}$ for $\mathbf{E}_{7}$, then $\Delta(1) \simeq \mathfrak{C}_{(2,3,4)}$;

- If $\Pi(1)=\left\{\alpha_{5}\right\}$ for $\mathbf{E}_{8}$, then $\Delta(1) \simeq \mathfrak{C}_{(2,3,5)}$.

This confirms Conjecture 5.3(i) for all these cases. The marked Dynkin diagrams for $\mathbf{D}_{5}$ and $\mathbf{E}_{n}$ are depicted below (the black node represents the simple root in $\Pi(1)$ ):

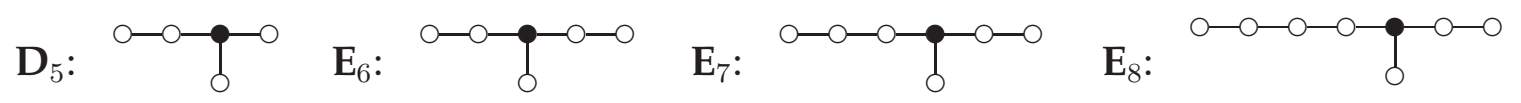

By the general rule, the subdiagram of white nodes represents $\mathfrak{g}(0)$, and the bonds through the black node determine the $\mathfrak{g}(0)$-module $\mathfrak{g}(1)$, see Remark 2.8.

Remark 5.8. An upper (lower) ideal in $\mathfrak{C}_{(k, m, n)}$ can be identified with a plane partition with at most $k$ rows, at most $m$ columns, and with each entry $\leqslant n$. Therefore, in case of $\mathcal{C}_{(k, m, n)}$, our $\mathcal{M}$-polynomial is nothing but the rank-generating function for such plane partitions. In [1, Theorem 11.2], one finds a closed formula for that generating function, which goes back to MacMahon. Letting $(t)_{r}=(1-t)\left(1-t^{2}\right) \ldots\left(1-t^{r}\right)$, one has

$$
\mathcal{M}_{\mathfrak{e}_{(k, m, n)}}=\frac{(t)_{1}(t)_{2} \ldots(t)_{k-1}(t)_{m+n}(t)_{m+n+1} \ldots(t)_{m+n+k-1}}{(t)_{m}(t)_{m+1} \ldots(t)_{m+k-1}(t)_{n}(t)_{n+1} \ldots(t)_{n+k-1}} .
$$

Substituting $t=1$, one obtains

$$
\# \mathfrak{A} \mathfrak{n}\left(\mathfrak{C}_{(k, m, n)}\right)=\frac{1 ! 2 ! \ldots(k-1) !(m+n) !(m+n+1) ! \ldots(m+n+k-1) !}{m !(m+1) ! \ldots(m+k-1) ! n !(n+1) ! \ldots(n+k-1) !} .
$$

The last formula appears in [5, p.553], where the relationship between plane partitions and antichains in $\mathcal{C}_{(k, m, n)}$ is also alluded to. In [13, p.81], one finds the rank-generating function for $\mathfrak{C}_{(k, m, n)}$ exactly in the form suggested by Eq. (5.1).

Remark 5.9. If the average value of the size of antichains in all $\mathfrak{X}_{\Delta(1)}$-orbits is the same, then it must be equal to the average value of the size of all antichains in $\Delta(1)$. By the very definition of $\mathcal{N}_{\Delta(1)}(t)$, the latter average value equals $\mathcal{N}_{\Delta(1)}^{\prime}(1) / \mathcal{N}_{\Delta(1)}(1)$. Therefore, if Conjecture 5.3(i),(ii) is true, then one must have the equality

$$
\frac{\mathcal{N}_{\Delta(1)}^{\prime}(1)}{\mathcal{N}_{\Delta(1)}(1)}=\frac{\# \Delta(1)}{d_{1}+1}
$$

Our previous calculations show that this is really the case for the abelian and extra-special gradings, see Eq. (3.1) and (4.6). 
Remark 5.10. If the average value of the size of upper ideals in all $\mathfrak{X}_{\Delta(1)}$-orbits is the same, then it must be equal to the average value of the size of all upper ideals in $\Delta(1)$. By the very definition of $\mathcal{M}_{\Delta(1)}(t)$, the latter average value equals $\mathcal{M}_{\Delta(1)}^{\prime}(1) / \mathcal{M}_{\Delta(1)}(1)$. Since $\mathcal{M}_{\Delta(1)}(t)$ is palindromic, the last fraction is equal to $\frac{1}{2} \operatorname{deg} \mathcal{M}_{\Delta(1)}(t)=\frac{1}{2} \operatorname{dim} \mathfrak{g}(1)$. This explains the average value in Conjecture 5.3(iii).

We proved that $\# \mathfrak{A} \mathfrak{n}(\Delta(1))=\# \Pi_{l} \cdot(h-1)$ in the extra-special case (Theorem 4.2(ii)). Combined with the conjectural value $\operatorname{ord}\left(\mathfrak{X}_{\Delta(1)}\right)=h-1$ and some explicit calculations, this suggests the following:

Conjecture 5.11. In the extra-special case, the number of $\mathfrak{X}_{\Delta(1)}$-orbits equals $\# \Pi_{l}$, and each orbit is of size $h-1$. Furthermore, if $h$ is even (which only excludes the case of $\mathbf{A}_{2 k}$, where $h=2 k+1$ ), then each $\mathfrak{X}_{\Delta(1)}$-orbit contains a unique Lagrangian upper ideal.

This conjecture is readily verified for $\mathbf{C}_{n}$ or $\mathbf{G}_{2}$, where $\# \Pi_{l}=1$, and the case of $\mathbf{A}_{n}$ is easy. It is also possible to perform all necessary calculations by hand for $\mathbf{F}_{4}$ and $\mathbf{E}_{6}$.

Our formulae for $\mathcal{N}$-polynomials in Sections 3 and 4 show that they are not always palindromic. Clearly, if $\mathcal{N}_{\mathcal{P}}(t)$ is palindromic, then it is monic, hence $\mathcal{P}$ has a unique antichain of maximal size. Therefore, if $\mathcal{P}$ is Sperner and $\mathcal{N}_{\mathcal{P}}(t)$ is palindromic, then $\mathcal{P}$ has a unique rank level of maximal size. It is plausible that, for the weight posets of the form $\Delta(1)$, this necessary condition is also sufficient:

Conjecture 5.12. Let $\mathfrak{g}=\bigoplus_{i \in \mathbb{Z}} \mathfrak{g}(i)$ be a 1-standard $\mathbb{Z}$-grading. Then $\mathcal{N}_{\Delta(1)}(t)$ is palindromic if and only if $\Delta(1)$ has a unique rank level of maximal size.

Our formulae confirm this conjecture in the abelian and extra-special cases.

Example 5.13. (Z-gradings versus periodic gradings)

1) For $\mathfrak{g}=\mathfrak{s o}_{8}$ (type $\mathbf{D}_{4}$ ), the extra-special $\mathbb{Z}$-grading corresponds to the branching node of the Dynkin diagram, i.e., $\Pi(1)=\left\{\alpha_{2}\right\}$. Hence $\widetilde{\mathfrak{g}(0)}=\left(\mathfrak{s l}_{2}\right)^{3}$ and $\mathfrak{g}(1)$ is the tensor product of the standard representations of three copies of $\mathfrak{s l}_{2}$. Therefore, $\Delta(1)$ is the 3dimensional Boolean algebra $\mathbb{B}^{3} \simeq \mathcal{C}_{2,2,2}$. Here $\# \mathfrak{A} \mathfrak{n}\left(\mathbb{B}^{3}\right)=20, \mathcal{H}_{\mathbb{B}^{3}}(t)=1+8 t+9 t^{2}+2 t^{3}$, and $h-1=5$. In fact, all the relevant assertions of the above conjectures are satisfied here. In particular, ord $\left(\mathfrak{X}_{\mathbb{B}^{3}}\right)=5, \mathfrak{A n}\left(\mathbb{B}^{3}\right)$ consists of four $\mathfrak{X}_{\mathbb{B}^{3}}$-orbits of size 5 , and both average values are constant, as prescribed.

On the other hand, $\mathfrak{s o}_{8}$ has a $\mathbb{Z}_{2}$-grading such that $\mathfrak{g}_{0}=\left(\mathfrak{s l}_{2}\right)^{4}$ and $\mathfrak{g}_{1}$ is the tensor product of the standard representations of four copies of $\mathfrak{s l}_{2}$. Hence $\Delta_{1}$ is the 4-dimensional Boolean algebra $\mathbb{B}^{4}$. The poset $\mathbb{B}^{4}$ has five rank levels and the corresponding level cardinalities $\#\left(\mathbb{B}^{4}\right)_{i}$ are $1,4,6,4,1$. Here the hypothetical product formula for $\# \mathfrak{A} \mathfrak{n}\left(\Delta_{1}\right)$, see Eq. (5.1), yields the output

$$
\frac{2^{1} 3^{4} 4^{6} 5^{4} 6^{1}}{1^{1} 2^{4} 3^{6} 4^{4} 5^{1}}=500 / 3=166,66 \ldots
$$


which is absurd. Furthermore, the hypothetical product formula for $\mathcal{M}_{\Delta_{1}}(t)$ gives a rational function that is not a polynomial. Thus, one cannot always expect the validity of Eq. (5.1) if $V$ is not associated with a $\mathbb{Z}$-grading.

It is known that $\# \mathfrak{A} \mathfrak{n}\left(\mathbb{B}^{4}\right)=168$ and the sizes of $\mathfrak{X}_{\mathbb{B}^{4}}$-orbits are $2,3,6$, see e.g. [7, p.73]. Therefore $\operatorname{ord}\left(\mathfrak{X}_{\mathbb{B}^{4}}\right)=6$, which agrees with Conjecture 5.3(i). But, the average value of sizes of antichains along $\mathfrak{X}_{\mathbb{B}^{4}}$-orbits is not constant here! Let us regard elements of $\mathbb{B}^{4}$ as subsets of $\{1,2,3,4\}$. For the orbit of $\Gamma_{1}=\{\varnothing\}$, the above average value is $8 / 3$, while for the orbit of $\Gamma_{2}=\{\{1,2\},\{3,4\}\}$, the average value is 3 . Furthermore, explicit computations show that

$$
\mathcal{N}_{\mathbb{B}^{4}}(t)=1+16 t+55 t^{2}+64 t^{3}+25 t^{4}+6 t^{5}+t^{6} .
$$

That is, whereas $\mathbb{B}^{4}$ has a unique rank level of maximal size, $\mathcal{N}_{\mathbb{B}^{4}}(t)$ is not palindromic.

2) The choice of the branching node $\alpha_{i}$ in the extended Dynkin diagram $\tilde{\mathbf{E}}_{n}(n=6,7,8)$ provides a $\mathbb{Z}_{d}$-grading with $d=\left[\theta: \alpha_{i}\right]$ such that $\mathfrak{g}_{0}=\mathfrak{s l}_{k} \times \mathfrak{s l}_{m} \times \mathfrak{s l}_{n}$ and $\mathfrak{g}_{1}=\mathcal{R}\left(\varpi_{1}\right) \otimes$ $\mathcal{R}\left(\varpi_{1}^{\prime}\right) \otimes \mathcal{R}\left(\varpi_{1}^{\prime \prime}\right)$. Therefore, $\Delta_{1} \simeq \mathcal{C}_{(k, m, n)}$. Here $(k, m, n)=(3,3,3),(2,4,4),(2,3,6)$ for $\mathbf{E}_{6}$, $\mathbf{E}_{7}, \mathbf{E}_{8}$, respectively, see pictures below. The respective values of $d$ are $3,4,6$.

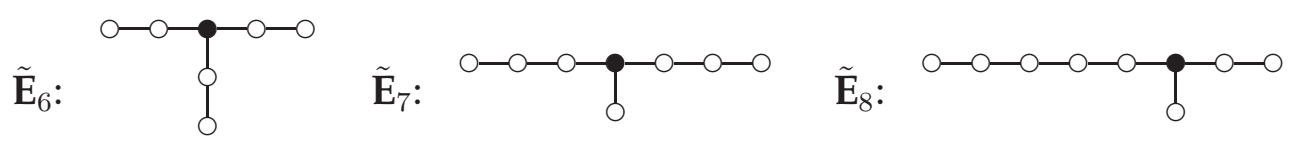

By the general rule, the subdiagram of white nodes represents $\mathfrak{g}_{0}$, and the bonds through the black node determine the $\mathfrak{g}_{0}$-module $\mathfrak{g}_{1}$, see [27, $\left.\S 8\right],[28$, Ch. $3, \S 3.7]$ for details.

As explained in Remark 5.8, Conjecture 5.1 (or Eq. (5.1)) does provide here the $\mathcal{M}-$ polynomial of $\Delta_{1}$. However, at least in the $\mathbf{E}_{6}$-case, one detects two $\mathfrak{X}_{\Delta_{1}}$-orbits with different values for the average size of antichains. Thus, Conjecture 5.3(ii) fails there.

Example 5.14. For the 1-standard $\mathbb{Z}$-grading of $\mathbf{E}_{8}$ with $\Pi(1)=\left\{\alpha_{8}\right\}$, we have $\widetilde{\mathfrak{g}(0)}=\mathbf{A}_{7}=$ $\mathfrak{s l}_{8}$ and $\mathfrak{g}(1)=\mathcal{R}\left(\varpi_{3}\right)$, the third fundamental representation of $\mathfrak{s l}_{8}$. Then $\operatorname{dim} \mathfrak{g}(1)=\left(\begin{array}{l}8 \\ 3\end{array}\right)=$ 56 and the poset $\Delta(1)=\mathcal{P}\left(\varpi_{3}\right)$ has 16 rank levels whose sizes are $1,1,2,3,4,5,6,6,6,6,5,4,3,2,1,1$. Here the formulae of Conjecture 5.1 provide a polynomial in $t$ with nonnegative coefficients and an integer! That is, conjecturally, it should be true that $\# \mathfrak{A} \mathfrak{n}(\Delta(1))=11 \cdot 13 \cdot 17=2431$ and $\operatorname{ord}(\mathfrak{X})=17$.

Concluding remarks. In this section, we formulated a bunch of conjectures for various properties of weight posets. I have every confidence in validity of all these conjectures for the weight posets of the form $\Delta(1)$. Moreover, some of the conjectures may have a wider range of applicability. For instance, the posets $\mathcal{C}_{(k, m, n)}$ are related to periodic or $\mathbb{Z}$ gradings only for a limited set of triples $(k, m, n)$. (All those instances essentially appear in Examples 5.7 and 5.13(2).) But the formula for the $\mathcal{M}$-polynomial in Conjecture 5.1 applies to all such posets, see Remark 5.8 . 
Furthermore, it is likely that, for all posets $\mathcal{P}=\mathcal{C}_{(k, m, n)}$, the order of $\mathfrak{X}_{\mathcal{P}}$ equals $k+m+$ $n-1$, which agrees with Conjecture 5.3(i). (At least, this is proved for $k=2$ in [5].)

On the other hand, the property on the average value of the size of antichains in $\mathfrak{X}$ orbits (see Conjecture 5.3(ii)) seems to be the most vulnerable one. Our examples suggest that it fails once we leave the variety of weight posets related to $\mathbb{Z}$-gradings.

Acknowledgements. This is an expanded version of my talk at the workshop "Non-crossing partitions" (Bielefeld, June 2014). I would like to thank the organisers for possibility to speak there. While preparing my talk, I dug up my old notes on the subject, made several new observations, and even produced some proofs.

\section{REFERENCES}

[1] G.E. ANDREWS. "The theory of partitions", Encyclopedia of Mathematics and its Applications, Vol. 2. Addison-Wesley Publishing Co., Reading, Mass.-London-Amsterdam, 1976. xiv+255 pp.

[2] D. Armstrong, C. Stump and H. Thomas. A uniform bijection between nonnesting and noncrossing partitions, Trans. Amer. Math. Soc., 365, no. 8 (2013), 4121-4151.

[3] N. BourbaKi. "Groupes et algèbres de Lie", Chapitres 4,5 et 6, Paris: Hermann 1975.

[4] N. BourbaKI. "Groupes et algèbres de Lie", Chapitres 7 et 8, Paris: Hermann 1975.

[5] P.J. CAMERON and D.G. FON-DER-FlaAss. Orbits of antichains revisited, Europ. J. Combinatorics, 16 (1995), 545-554.

[6] P. CelLini and P. PAPI. ad-nilpotent ideals of a Borel subalgebra II, J. Algebra, 258 (2002), 112-121.

[7] M. DeZA and K. FuKUDA. Loops of clutters, in: "Coding theory and design theory", Part 1, pp. 72-92 (The IMA volumes in Mathematics and its Appl., 20), Springer-Verlag, 1990.

[8] Е.Б. Дынкин. Некоторые свойства системы весов линейного представления полупростой группы Ли, ДАН, т. 71, № 3 (1950), 221-224. (Russian) (= E.B. DyNKIN. Some properties of the system of weights of a linear representation of a semisimple Lie group, Doklady Akad. Nauk SSSR, 71 (1950)).

[9] D.G. FON-DER-FLAASS. Orbits of antichains in ranked posets, Europ. J. Combinatorics, 14 (1993), 17-22.

[10] R. Howe. Perspectives on Invariant Theory: Schur duality, multiplicity-free actions and beyond, The Schur lectures (1992), 1-182, Israel Math. Conf. Proc., 8, Bar-Ilan Univ., Ramat Gan, 1995.

[11] J.E. Humphreys. "Reflection Groups and Coxeter Groups", Cambridge Univ. Press, 1992.

[12] I. Macdonald. The Poincaré series of a Coxeter group, Math. Annalen, 199 (1972), 161-174.

[13] I. MacdonALD. "Symmetric functions and Hall polynomials", 2nd edition. The Clarendon Press, Oxford, 1995. $\mathrm{x}+475 \mathrm{pp}$.

[14] D. Panyushev. On orbits of antichains of positive roots, European J. Combin., 30, no. 2 (2009), 586-594.

[15] D. PANYUSHEV. Properties of weight posets for weight multiplicity free representations, Moscow Math. J., 9, no. 4 (2009), 867-883.

[16] D. PANYUSHEv. Two covering polynomials of a finite poset, with applications to root systems and ad-nilpotent ideals, J. Combinatorics, 3, no. 1 (2012), 63-89 (= math.C0/0502386, v4, 23 pp.)

[17] D. PANYUSHEV. On Lusztig's $q$-analogues of all weight multiplicities of a representation, (arXiv: [math.RT] 1406.1453, 15 pp.)

[18] P. PAPI. A characterization of a special ordering in a root system, Proc. Amer. Math. Soc., 120 (1994), 661-665. 
[19] R. Proctor. Representations of $\mathfrak{s l}(2, \mathbb{C})$ on posets and the Sperner property, SIAM J. Alg. Disc. Meth., 3, no. 2 (1982), 275-280.

[20] R. Proctor. Classical Bruhat orders and lexicographic shellability. J. Algebra, 77, no. 1 (1982), 104-126.

[21] R. Proctor. Bruhat lattices, plane partition generating functions, and minuscule representations, $E u-$ ropean J. Combin., 5, no. 4 (1984), 331-350.

[22] B. SAGAN. The cyclic sieving phenomenon: a survey. Surveys in combinatorics 2011, 183-233, London Math. Soc. Lecture Note Ser., 392, Cambridge Univ. Press, Cambridge, 2011.

[23] D. RuSH and X. SHI. On orbits of order ideals of minuscule posets, J. Algebraic Combin., 37, no. 3 (2013), 545-569.

[24] J. STEMBRIDGE. On minuscule representations, plane partitions and involutions in complex Lie groups, Duke math. J., 73 (1994), 469-490.

[25] R. Suter. Coxeter and dual Coxeter numbers, Comm. Alg., 26 (1998), 147-153.

[26] R.P. Stanley. “Enumerative Combinatorics", vol. 1. Cambridge Univ. Press, 1997.

[27] Э.Б. ВинБеРг. Группа Вейля градуированной алгебры Ли, Изв. АН СССР. Сер. Матем. 40, № 3 (1976), 488-526 (Russian). English translation: E.B. VinberG. The Weyl group of a graded Lie algebra, Math. USSR-Izv. 10 (1976), 463-495.

[28] Э.Б. ВиньеРГ, В.В. ГОРБАцеВИЧ, А.Л. ОНИщик. “Группы и алгебры Ли - 3”, Соврем. пробл. математики. Фундам. направл., т. 41. Москва: ВИНИТИ 1990 (Russian). English translation: V.V. Gorbatsevich, A.L. OnishchiK and E.B. VinberG. "Lie Groups and Lie Algebras III" (Encyclopaedia Math. Sci., vol. 41) Berlin: Springer 1994.

INSTITUTE FOR INFORMATION TRANSMISSION PROBLEMS OF THE R.A.S.,

B. KARETNYi PER. 19, MOSCOW 127994, RUSSIA

E-mail address: panyushev@iitp.ru

INDEPENDENT UNIVERSITY OF MOSCOW, BOL'SHOI VLASEVSKII PER. 11, 119002 MOSCOW, RussiA 